J.-Y. CHEMIN

\title{
Interaction contrôlée dans les équations aux dérivées partielles non linéaires
}

Bulletin de la S. M. F., tome 116, no 3 (1988), p. 341-383

<http://www.numdam.org/item?id=BSMF_1988_116_3_341_0>

(C) Bulletin de la S. M. F., 1988, tous droits réservés.

L'accès aux archives de la revue «Bulletin de la S. M. F. » (http: //smf.emath.fr/Publications/Bulletin/Presentation.html) implique l'accord avec les conditions générales d'utilisation (http://www.numdam.org/ conditions). Toute utilisation commerciale ou impression systématique est constitutive d'une infraction pénale. Toute copie ou impression de ce fichier doit contenir la présente mention de copyright.

\section{Numdam}




\title{
INTERACTION CONTRÔLÉE DANS LES ÉQUATIONS AUX DÉRIVÉES PARTIELLES NON LINÉAIRES
}

PAR

\author{
J.-Y. CHEMIN $\left({ }^{*}\right)$
}

RÉSumÉ. - Avec un calcul symbolique bilinéaire de type paradifférentiel, on démontre, pour une solution d'une équation aux dérivées partielles non linéaires strictement hyperbolique, une majoration de son front d'onde relatif à l'espace de Sobolev $H^{\sigma}$, à partir de sa connaissance dans le passé, $\sigma$ allant jusqu'au triple de la régularité globale de la solution.

ABstract. - With a bilinear symbolic calculus of paradifferential type, we prove, for a solution of a strictly hyperbolic non linear partial differential equation, an estimate of its $H^{\sigma}$-wave front, $\sigma$ going up to three times the global regularity of the solution.

\section{Introduction}

0.0. Nature du problème. - On cherche à décrire la régularité microlocale d'une solution réelle $u \in H_{\text {loc }}^{s}(\Omega), s$ étant suffisamment grand, et $\Omega$ étant un ouvert de $\mathbb{R}^{n}$, de l'équation

$$
f\left(x, u, \partial^{\alpha} u\right)_{|\alpha| \leq m}=0 \quad \text { sur } \Omega
$$

où $f$ est une fonction $\mathcal{C}^{\infty}$ de ses arguments. On supposera dans tout ce travail que $p_{m}(x, \xi)=\sum_{|\alpha|=m} \partial f / \partial u_{\alpha}\left(x, u, \partial^{\beta} u\right)_{|\beta| \leq m} \xi^{\alpha}$ est un polynôme strictement hyperbolique par rapport à $\xi_{1}$ et que l'on a une situation d'évolution, c'est-à-dire que toute bicaractéristique de $p_{m}$ issue d'un point de $\Omega_{+}=\Omega \cap\left\{x_{1}>0\right\}$ coupe $\Omega_{-}=\Omega \cap\left\{x_{1}<0\right\}$.

J.M. Bony a démontré dans [6], grâce à la construction du calcul paradifférentiel, que les singularités microlocales $H^{\sigma}$, pour $\sigma \leq 2 s-s_{0}$, avaient un comportement linéaire vis à vis de la géométrie des bicaractéristiques

(*) Texte reçu le 8 janvier 1987 , révisé le 11 juin 1987 .

J.-Y. Chemin, École Polytechnique, Centre de Mathématiques, 91128 Palaiseau Cedex, France.

BULLETIN DE LA SOCIÉTÉ MATHÉMATIQUE DE FRANCE 0037-9484/1988/341/ $\$ 5.00$

(C) Société mathématique de France 
de $p_{m}$. De nombreux résultats s'affranchissant de cette limitation ont été obtenus, d'une part sous des hypothèses de régularité conormale par rapport à une ou plusieurs hypersurfaces par S. AlinhaC (voir [1], [2], [3]) par J.M. Bony (voir [7] et [8]), et par R. Melrose et N. RitTer (voir [13]), d'autre part, en dimension 2 par J. RAUCH et M. ReED dans [16], par P. Godin dans [12], ainsi que dans [9] et [10].

On s'intéresse dans ce travail au phénomène de la propagation de la régularité jusqu'à $3 s-2 s_{0}$, qui a été observé pour la première fois par M. BEALS dans [4], où il démontrait que si $u$ est solution $H^{s}$ de

$$
\frac{\partial^{2}}{\partial x_{1}^{2}} u-\sum_{i=2}^{n} \frac{\partial^{2}}{\partial x_{i}^{2}} u=f(x, u)
$$

avec $s \geq s_{0}$ et $f$ polynomiale en $u$, la régularité se propageait jusqu'à l'ordre $3 s-2 s_{0}$ et que ce résultat était essentiellement optimal. Puis, dans [5], il en donnait une généralisation au cas de l'équation $P u=$ $f(x, u, \partial u)$, l'opérateur $P$ étant un opérateur strictement hyperbolique d'ordre 2 .

On se propose ici de démontrer, pour une solution réelle $H^{s}$ d'une équation (E) strictement hyperbolique, $s \geq s_{0}$, un théorème général de régularité donnant une majoration de son front d'onde $H^{\sigma}$ à partir de la connaissance de ses directions caractéristiques dans un domaine d'influence, avec $\sigma \leq 3 s-2 s_{0}$. Ce théorème contient la conjecture faite dans [5] sur la propagation des singularités jusqu'à l'ordre $H^{3 s-2 s_{0}}$ pour les équations quasi-linéaires strictement hyperboliques d'ordre 2 .

On peut introduire, pour une solution $u$ d'une équation (E) strictement hyperbolique, la notion d'hypersurface lente, c'est-à-dire d'hypersurface caractéristique telle qu'en tout point $(x, \xi)$ de son conormal, l'hyperplan tangent en $\xi$ à $\operatorname{Car} p_{m \mid x}$ ne recoupe $\operatorname{Car} p_{m \mid x}$ qu'en $\mathbb{R}^{*} \xi$. Remarquons que ceci est toujours le cas à l'ordre 2 .

Le théorème général de régularité assure que, si $\Sigma$ est une hypersurface lente, $u$ étant solution de l'équation (E) supposée strictement hyperbolique, si le front d'onde $H^{\sigma}$ de $u$ a ses directions caractéristiques dans le conormal de $\Sigma$ dans le passé, il en est de même dans l'avenir. C'est le phénomène d'évolution d'une onde lente; on donnera également une illustration géométrique de l'interaction de deux tels phénomènes.

De plus, on exhibera une solution $u$ de

$$
\left(\frac{\partial^{2}}{\partial t^{2}}-c_{1}^{2} \frac{\partial^{2}}{\partial x^{2}}-c_{1}^{2} \frac{\partial^{2}}{\partial y^{2}}\right)\left(\frac{\partial^{2}}{\partial t^{2}}-c_{2}^{2} \frac{\partial^{2}}{\partial x^{2}}-c_{2}^{2} \frac{\partial^{2}}{\partial y^{2}}\right) u=\beta(t) u^{2},
$$

où $c_{1}>c_{2}$ et $\beta \in \mathcal{C}_{0}^{\infty}\left(\mathbb{R}_{+}\right)$, telle que son front d'onde dans le passé soit inclus dans le conormal de cône rapide $C_{1}=\left\{x^{2}+y^{2}=c_{1}^{2} t^{2}\right\}$ et telle que, dans l'avenir, $u$ ne soit pas $H^{2 s+s_{1}}$ sur $\left\{x^{2}+y^{2} \in\left[c_{2}^{2} t^{2}, c_{1}^{2} t^{2}\right]\right\}$.

TOME $116-1988-\mathrm{N}^{\circ} 3$ 
Ceci montre la dissymétrie entre la propagation des ondes lentes et celles des ondes rapides.

Cet exemple montre le caractère essentiellement optimal du théorème général de régularité que l'on peut résumer par le schéma suivant.

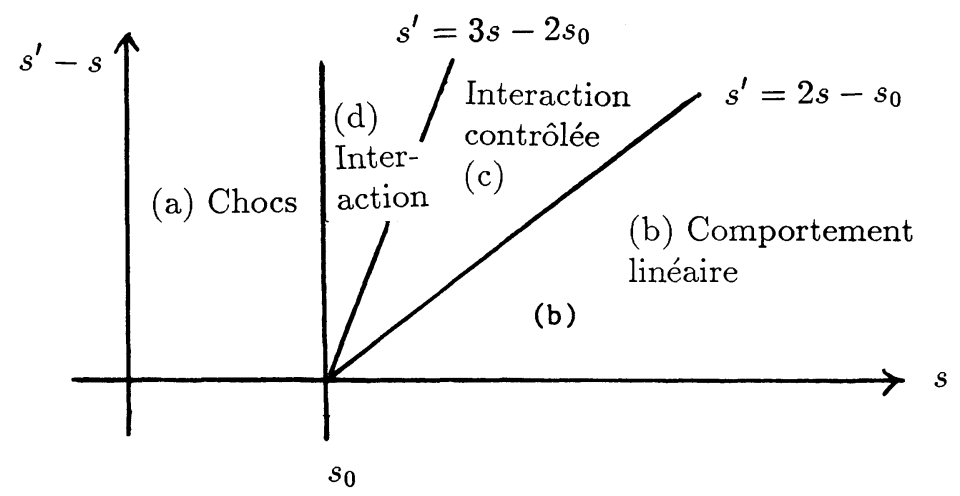

L'obtention de ce théorème nécessitera la transformation de l'équation non linéaire étudiée, non plus en une équation paradifférentielle linéaire modulo un reste $H^{2 s-s_{1}}$ comme dans [6] et [14], mais en une équation paradifférentielle bilinéaire modulo un reste $H^{3 s-2 s_{1}}$.

Pour démontrer que la partie bilinéaire du reste a la régularité microlocale nécessaire, il faut construire un calcul symbolique de type paradifférentiel bilinéaire, et l'appliquer par le biais du lemme de l'hyperplan tangent 3.3.

0.1. Notations et rappels. - Soit $F$ un espace normé, et si $x \in F$ on note $\langle x\rangle=\left(1+\|x\|_{F}^{2}\right)^{1 / 2}$. Dans ce travail, nous utiliserons la théorie de Littlewood-Paley; on appelle partition de l'unité dyadique la donnée d'une couronne $C$ de $\mathbb{R}^{n}$ et d'une fonction $\varphi$ de $\mathcal{C}_{0}^{\infty}(C)$ telle que la fonction $\psi(\xi)=1-\sum_{q=0}^{+\infty} \varphi\left(2^{-q} \xi\right)$ soit à support compact. Pour $u \in \mathcal{S}^{\prime}\left(\mathbb{R}^{n}\right)$ on a :

$$
\psi(D) u+\sum_{q=0}^{+\infty} \varphi\left(2^{-q} D\right) u=u .
$$

On désignera $\varphi\left(2^{-q} D\right) u$ par $\Delta_{q}(u)$ ou bien par $u_{q}$ lorsqu'il n'y aura pas d'ambiguïté, $\psi(D) u$ par $\Delta_{-1}(u)$ ou bien par $u_{-1}$ et $\psi\left(2^{-q} D\right) u$ par $S_{q}(u)$ (remarquons que l'on a $S_{q}(u)=\sum_{p=-1}^{q-1} u_{p}$ ).

On supposera connues les diverses caractérisations de l'espace de Sobolev $H^{s}$ et de l'espace de Hölder $C^{\rho}$ par cette théorie. (Voir [6]). On pose $H_{-}^{s}=\bigcap_{\epsilon>0} H^{s-\epsilon}$. 
On note $|\cdot|_{s}\left(\right.$ resp. $\|\cdot\|_{\rho}$ ) la norme dans $H^{s}$ (resp. dans $C^{\rho}$ pour $\rho \neq 0$ ); on note $\|\cdot\|_{0}$ la norme dans $L^{\infty}$. Étant donnés une partition de l'unité dyadique, et un entier positif $N_{0}$ tel que $2^{-N_{0}} \operatorname{Supp} \psi+C$ soit une couronne, on appellera paraproduit l'application suivante de $\mathcal{S}^{\prime} \times \mathcal{S}^{\prime}$ dans $\mathcal{S}^{\prime}$ :

$$
T_{u} v=\sum_{q} S_{q-N_{0}}(u) \cdot v_{q}
$$

On notera encore $T$ l'application suivante de $\mathbb{R}^{2 n}$ dans $\mathbb{R}$ :

$$
T(\xi, \eta)=\sum_{q} \psi\left(2^{-q+N_{0}} \xi\right) \varphi\left(2^{-q} \eta\right)
$$

Lorsque, par exemple, $u$ et $v$ sont dans $L^{2}$, on a

$$
\mathcal{F}\left(T_{u} v\right)(\xi)=\frac{1}{(2 \pi)^{n}} \int T(\xi-\eta, \eta) \hat{u}(\xi-\eta) \hat{v}(\eta) d \eta
$$

On supposera connues les propriétés opératoires du paraproduit dans les $H^{s}$ et les $C^{\rho}$ (voir [6]).

Si $u \in \mathcal{D}^{\prime}(\Omega), W F(u)$ (resp. $W F_{\sigma}(u)$ ) désigne le front d'onde (resp. le front d'onde $\left.H^{\sigma}\right)$ de $u$.

Lorsque $A \in \mathrm{Op} S^{m}(\Omega)$, l'ensemble des opérateurs pseudodifférentiels classiques sur $\Omega, \mathrm{SE}(A)$ désigne le support essentiel de $A$.

Soit $\rho$ un réel strictement positif non entier, on désignera par $\sum_{\rho}^{m}(\Omega)$ l'ensemble des $\ell(x, \xi)$ somme, pour $j$ variant de 0 à $[\rho]$, de $\ell_{j}(x, \xi)$ telles que $\ell_{j}$ soit homogène en $\xi$ de degré $m-j$ pour $|\xi| \geq 1$, et que, pour tout $\varphi \in \mathcal{C}_{0}^{\infty}(\Omega)$,

$$
\left\|\partial_{\xi}^{\alpha} \varphi \ell_{j}(\cdot, \xi)\right\|_{\rho-j} \leq C\langle\xi\rangle^{m-j-|\alpha|}
$$

et par $\operatorname{Op} \sum_{\rho}^{m}(\Omega)$ l'ensemble des opérateurs paradifférentiels associés; pour les définitions complètes et les propriétés opératoires nous renvoyons à $[6]$.

De plus, dans ce travail, on dira qu'un espace de fonctions $A$ est une algèbre si et seulement si pour toute $u$ et $v$ dans $A$ et toute $f, \mathcal{C}^{\infty}$ au voisinage des valeurs de $u, u v \in A$ et $f(u) \in A$.

\section{1. Énoncé des résultats}

L'énoncé du théorème de régularité 1.2 nécessite la définition préalable des ensembles prenant en compte la propagation et l'interaction des singularités.

On suppose donc donnée une solution $u \in H_{\text {loc }}^{s}(\Omega)$ et réelle de l'équation ( $\mathrm{E}$ ) avec $s>\frac{1}{2} n+m+1-d$, l'entier $d$ valant 2 si l'équation ( $\mathrm{E}$ )

TOME $116-1988-\mathrm{N}^{\circ} 3$ 
est semi-linéaire, valant 1 si elle est quasi-linéaire et 0 sinon. Remarquons que si (E) est semi-linéaire, on peut supposer que la partie linéaire est pseudodifférentielle; dans ce cas, il faut supposer que $p_{m}(x,-\xi)=$ $(-1)^{m} p(x, \xi)$.

Définition 1.1. - On notera $F_{\sigma}(u)$ (ou $F_{\sigma}$ lorsqu'il n'y aura pas d'ambiguïté) la réunion des bicaractéristiques tournées vers l'avenir issues de points de $W F_{\sigma}(u) \cap \operatorname{Car} p_{m} \cap\left\{x_{1}<0\right\}$.

Remarquons que, d'après le théorème de propagation de Bony démontré dans [6], si $u$ solution de (E) sur un ouvert $\Omega$ de $\mathbb{R}^{n}$, alors, pour tout $\sigma \leq 2 s-\frac{1}{2} n-m+d-1, F_{\sigma}=W F_{\sigma}(u) \cap \operatorname{Car} p_{m}$.

Il est bien connu que deux singularités de directions non opposées interagissent par enveloppe convexe; pour $\sigma \leq 3 s-2 s_{0}$, M. BEALS a démontré dans certains cas que deux singularités de direction opposée interagissent dans l'hyperplan tangent à la variété caractéristique (voir [4] et [5]). Cela conduit à définir $\widetilde{G}_{\sigma}$ où $\left.\widetilde{G}_{\sigma}\right|_{x}$ est la réunion des ensembles $F_{\left.\sigma_{1}\right|_{x}}+\left.F_{\sigma_{2}}\right|_{x}$ lorsque $\left(\sigma_{1}, \sigma_{2}\right)$ parcourt l'ensemble des couples tels que $\sigma_{1}+\sigma_{2}=\sigma+\frac{1}{2} n$ et des plans tangents en $\xi$ à Car $\left.p_{m}\right|_{x}$ lorsque $\xi$ parcourt $F_{\sigma / 2+n /\left.4\right|_{x}}$. De façon plus concise on a :

$$
\widetilde{G}_{\sigma}=\bigcup_{\sigma_{1}+\sigma_{2}=\sigma+n / 2}\left(F_{\sigma_{1}}+F_{\sigma_{1}}\right) \cup T_{F_{\sigma / 2}+n / 4} \operatorname{Car} p_{m} .
$$

Malheureusement, cet ensemble est légèrement trop petit.

Définition 1.2.

(i) On désigne par $G_{\sigma}^{(1)}$ l'ensemble des $(x, \xi)$ dans $T^{*} \Omega$ tels qu'il existe $\xi_{1}$ et $\xi_{2}$ de directions non opposées, tels que $\xi \in \mathbb{R}_{+} \xi_{1}+\mathbb{R}_{+} \xi_{2}$ et tels que, pour tout $\left(\sigma_{1}, \sigma_{2}\right)$ tel que $\sigma_{1}+\sigma_{2}=\sigma+\frac{1}{2} n$, avec $\left.\xi_{1} \in F_{\sigma_{1}}\right|_{x}$ ou $\left.\xi_{2} \in F_{\sigma_{2}}\right|_{x}$.

(ii) On désigne par $G_{\sigma}^{(2)}$ l'ensemble des $(x, \xi)$ dans $T^{*} \Omega$ tels qu'il existe $\eta$ dans $F_{\sigma / 2+n / 4}$ tel que $\left.\xi \in T_{\eta} \operatorname{Car} p_{m}\right|_{x}$.

(iii) On pose enfin $G_{\sigma}=G_{\sigma}^{(1)} \cup G_{\sigma}^{(2)}$.

Remarquons que $\widetilde{G}_{\sigma} \subset G_{\sigma} \subset \bigcap_{\epsilon>0} \widetilde{G}_{\sigma+\epsilon}$. Le théorème ci-dessous fournit une première justification à cette définition.

THÉorème 1.1. - Si $(\mathrm{E})$ est semi linéaire, si $v$ solution $H_{\mathrm{loc}}^{s}(\Omega)$, $s>\frac{1}{2} n+m-1$ de $P_{m} v=0$, telle que, pour tout $\sigma \leq 3 s-n-m+1$, $W F_{\sigma}(v) \subset F_{\sigma}(v)$, alors, pour tout $\sigma \leq 3 s-n-m+1$ et toute $g \in \mathcal{C}^{\infty}$ on $a W F_{\sigma} g(v) \subset G_{\sigma}$

Les singularités créées par interaction dans les $G_{\sigma}$ vont à leur tour se propager; d'où la définition suivante : 
Définition 1.3. - $H_{\sigma}$ désigne la réunion des bicaractéristiques tournées vers l'avenir issues des points de $G_{\sigma} \cap \operatorname{Car} p_{m}$.

On peut maintenant énoncer le théorème général de régularité :

THÉORÈmE 1.2. - Si u est solution $H_{\text {loc }}^{s}(\Omega)$ réelle de $(\mathrm{E})$ avec $s>\frac{1}{2} n+m+1-d$, alors pour tout $\sigma \leq 3 s-n-2 m-2+2 d$

$$
\begin{aligned}
& W F_{\sigma}(u) \cap \operatorname{Car} p_{m} \subset F_{\sigma} \cup H_{\sigma+m+1-d} \\
& W F_{\sigma+1}(u) \cap\left(\operatorname{Car} p_{m}\right)^{c} \subset G_{\sigma+m+1-d} .
\end{aligned}
$$

Avant de démontrer les ThÉorÈmes 1.1 et 1.2, ce qui sera l'objet des paragraphes 2 et 3 , nous allons en donner divers corollaires et illustrations.

Intéressons nous dans un premier temps aux équations d'ordre 2 :

Corollaire 1.1. - Soit u solution réelle $H_{\mathrm{loc}}^{s}(\Omega)$ de $(\mathrm{E})$, supposée d'ordre $2, s>\frac{1}{2} n+3-d$, pour tout $\sigma \leq 3 s-n-6+2 d$, si $(x, \xi)$ est un point caractéristique de $\rho$, et si $\Gamma$ désigne la bicaractéristique issue de $(x, \xi)$ alors : si u est microlocalement $H^{\sigma}$ en $(x, \xi)$, alors $u$ l'est aussi tout le long de $\Gamma$.

Le corollaire résulte du théorème par le fait que Car $\left.p_{2}\right|_{x}$ est convexe à cause de la stricte hyperbolicité, donc si $\tau \leq 3 s-3-n+d ; G_{\tau} \cap \operatorname{Car} p_{2}=\emptyset$. Remarquons que ce corollaire contient les résultats et la conjecture de [5].

Pour donner quelques illustrations géométriques au THÉORÈme 1.2, on pose la définition suivante.

Définition 1.4 .

(i) Soit $\Sigma$ une hypersurface caractéristique pour $p_{m} \in \Sigma_{\rho}^{m}(\Omega), \rho>1$, $p_{m}$ strictement hyperbolique homogène de degré $m$. On dit que $\Sigma$ est lente, si on a, pour tout $(x, \xi) \in N^{*} \Sigma$,

$$
\left.T_{\xi}\left(\left.\operatorname{Car} p_{m}\right|_{x}\right) \cap \operatorname{Car} p_{m}\right|_{x}=\mathbb{R}^{*} \xi
$$

(ii) On appelle onde lente d'ordre $\sigma$ une distribution $u$ telle que :

$$
\left\{\begin{array}{l}
\operatorname{Car} p_{m} \cap W F_{\sigma}(u) \subset N^{*} \Sigma \\
\left(\operatorname{Car} p_{m}\right)^{c} \cap W F_{\sigma+1}(u) \subset T_{N^{*} \Sigma} \operatorname{Car} p_{m}
\end{array}\right.
$$

avec $T_{N^{*} \Sigma} \operatorname{Car} p_{m}=\left\{(x, \xi) \in T^{*} \Omega\left|\exists \eta \in N^{*}\right|_{\left.\right|_{x}} ;\left.\xi \in T_{\eta} \operatorname{Car} p_{m}\right|_{x}\right\}$.

L'expression hypersurface lente provient du fait suivant : soit $P_{4}=$ $\square_{1} \square_{2}$ avec $c_{1}>c_{2}$. Soit $\Sigma$ une hypersurface caractéristique pour $\square_{2}$; elle est lente au sens de (i).

On va maintenant énoncer deux corollaires, l'un décrivant l'évolution d'une onde lente, l'autre l'interaction de plusieurs ondes lentes.

TOME $116-1988-\mathrm{N}^{\mathrm{O}} 3$ 
Corollaire 1.2. - Soient $u$ solution réelle $H_{\mathrm{loc}}^{s}(\Omega)$ de $(\mathrm{E}), s>$ $\frac{1}{2} n+m+1-d, \Sigma$ une hypersurface lente, $\sigma \leq 3 s-2 m-n-2+2 d$. Si $u$ est une onde lente d'ordre $\sigma$ dans le passé, elle l'est dans l'avenir.

Corollaire 1.3. - Soient $u$ solution réelle $H_{\mathrm{loc}}^{s}(\Omega)$ de $(\mathrm{E})$ avec $s>\frac{1}{2} n+m+1-d,(\Sigma)=\left(\Sigma_{i}\right)_{i \leq i \leq N}$ une famille de $N$ hypersurfaces lentes ne se coupant pas dans le passé. On suppose que $u$ est une onde lente d'ordre $\sigma$ par rapport à $\Sigma_{i}$ dans le passé, et ce pour tout $i \in\{1, \ldots, N\}$. Si $\sigma \leq 3 s-2 m-n+2 d-2$, alors :

$$
\begin{gathered}
W F_{\sigma}(u) \cap \operatorname{Car} p_{m} \subset \bigcup_{i=1}^{N} N^{*}\left(\Sigma_{i}\right) \cup H_{\sigma+m+1-d} \\
W F_{\sigma+1}(u) \cap\left(\operatorname{Car} p_{m}\right)^{c} \subset\left(\bigcup_{i=1}^{m} T_{N^{*} \Sigma_{i}}\left(\operatorname{Car} p_{m}\right)\right) \cap\left(\operatorname{Car} p_{m}\right)^{c} .
\end{gathered}
$$

Nous allons maintenant illustrer ces deux corollaires dans le cas de l'équation des ondes semi-linéaires à deux vitesses.

Considérons $\square_{i}=\partial_{t}^{2}-c_{i}^{2} \Delta$ avec $c_{1}>c_{2}$ et l'équation

$$
\square_{1} \square_{2}=f\left(x, u, \partial^{\beta} u\right)_{|\beta| \leq 3} .
$$

Soit $C_{i}$ le cône d'onde associé à $\square_{i}$ issu de 0 . D'après le Corollaire 1.3, si $u$ solution de $\left(\mathrm{E}^{\prime}\right), u$ réelle et $H_{\mathrm{loc}}^{s}$ avec $s>\frac{1}{2} n+3$, est une onde lente d'ordre $\sigma \leq 3 s-n-6$ par rapport à $C_{2}$ dans le passé, elle l'est aussi dans l'avenir. Par contre, si $W F(u) \subset N^{*}\left(C_{1}\right)$ dans le passé on a dans l'avenir la configuration de la Figure 1.

Nous montrerons au paragraphe 4, par le biais d'un exemple, que ce phénomène se produit effectivement.

Pour illustrer le Corollaire 1.3, on va considérer l'exemple suivant : $u$ solution de $\square_{1} \square_{2} u=f\left(x, u, \partial u, \partial^{2} u, \partial^{3} u\right)=0, u \in H_{\text {loc }}^{s}\left(\mathbb{R}^{3}\right)$ avec $s>\frac{1}{2} n+3$. On suppose, par exemple, que $u$ est une onde lente d'ordre $\sigma$ par rapport aux deux cônes lents issus de $(0,-a, 0)$ et $(0, a, 0)$ sur l'ouvert $] 0, \frac{a}{c_{2}}\left[\times \mathbb{R}^{2}\right.$; on représente alors le phénomène par le film suivant, qui dessine les singularités à $t=\mathrm{C}^{\text {te }}$. 


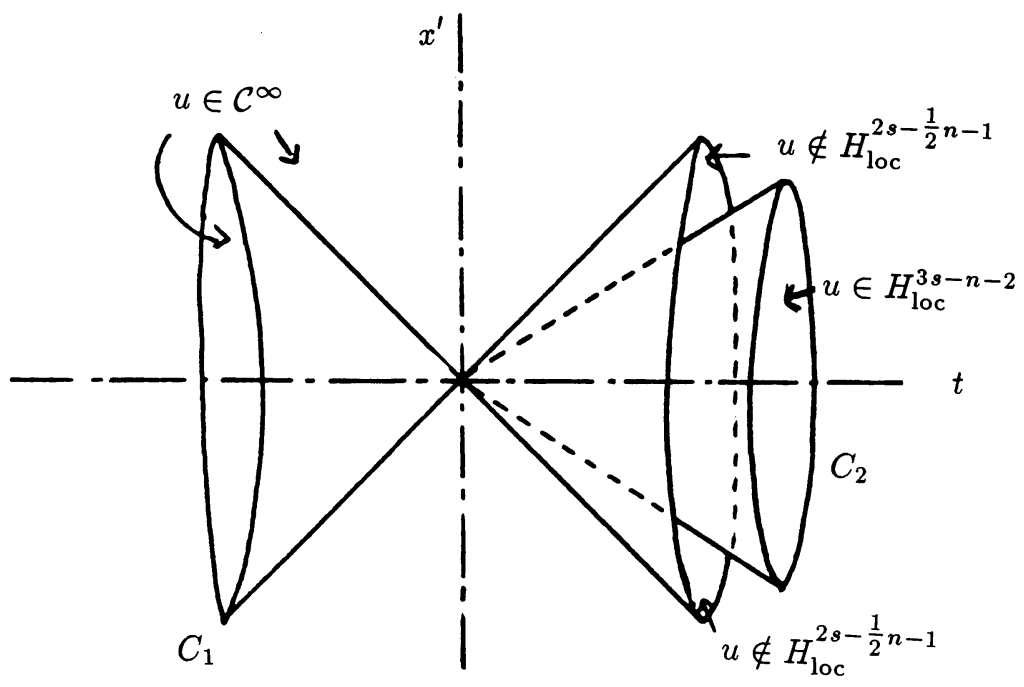

Fig. 1

$t<\frac{a}{c_{2}} \quad$ rien ne se passe $\quad t=\frac{a}{c_{2}} \quad$ c'est le début de l'interaction

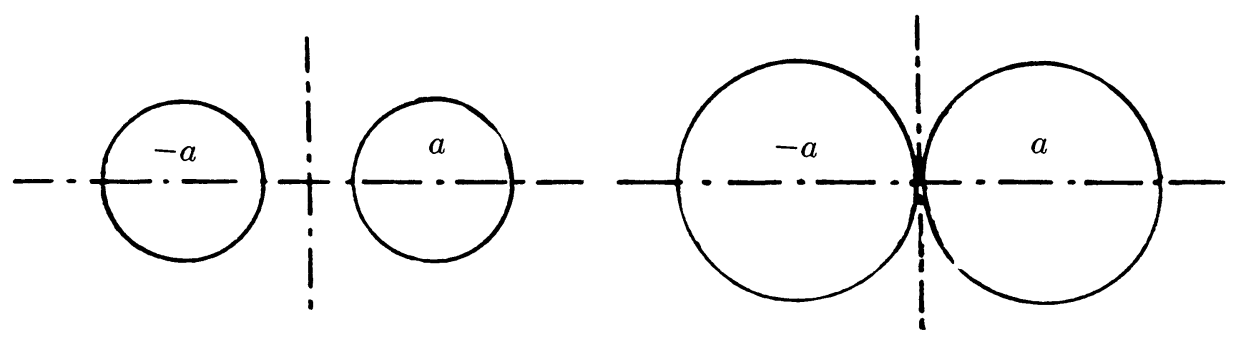

$$
\frac{a}{c_{2}}<t<\frac{a}{c_{2}} \frac{c_{1}}{\left(c_{1}^{2}-c_{2}^{2}\right)^{\frac{1}{2}}}
$$

TOME $116-1988-\mathrm{N}^{\mathrm{O}} 3$ 
Les singularités créées par interaction se propagent rapidement

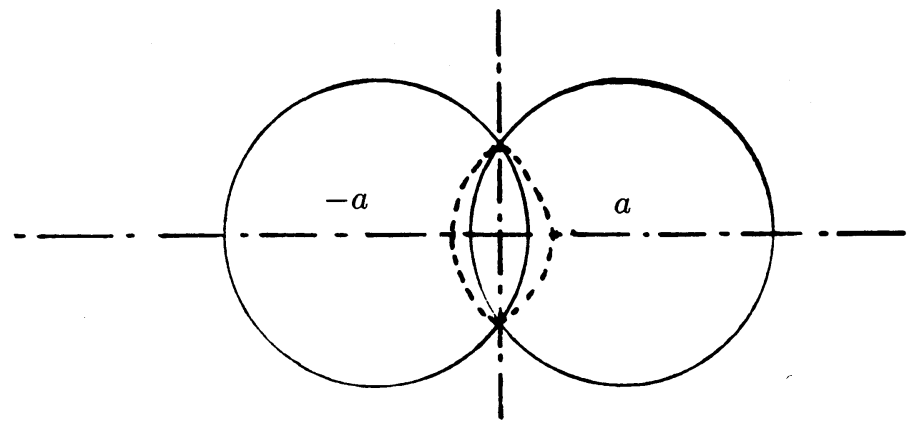

$t=\frac{a}{c_{2}} \frac{c_{1}}{\left(c_{1}^{2}-c_{2}^{2}\right)^{1 / 2}}, \quad$ c'est la fin de l'interaction.

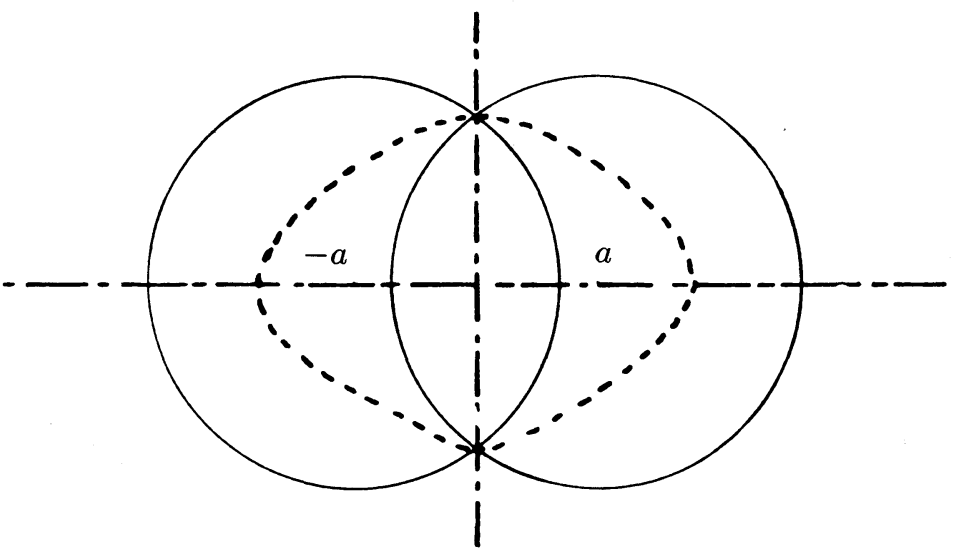

Pour $t>\frac{a c_{1}}{c_{2}\left(c_{1}^{2}-c_{2}^{2}\right)^{1 / 2}}$, chaque type de singularité se propage à sa BULLETIN DE LA SOCIÉTÉ MATHÉMATIQUE DE FRANCE 
vitesse, il n'y a plus d'interaction.

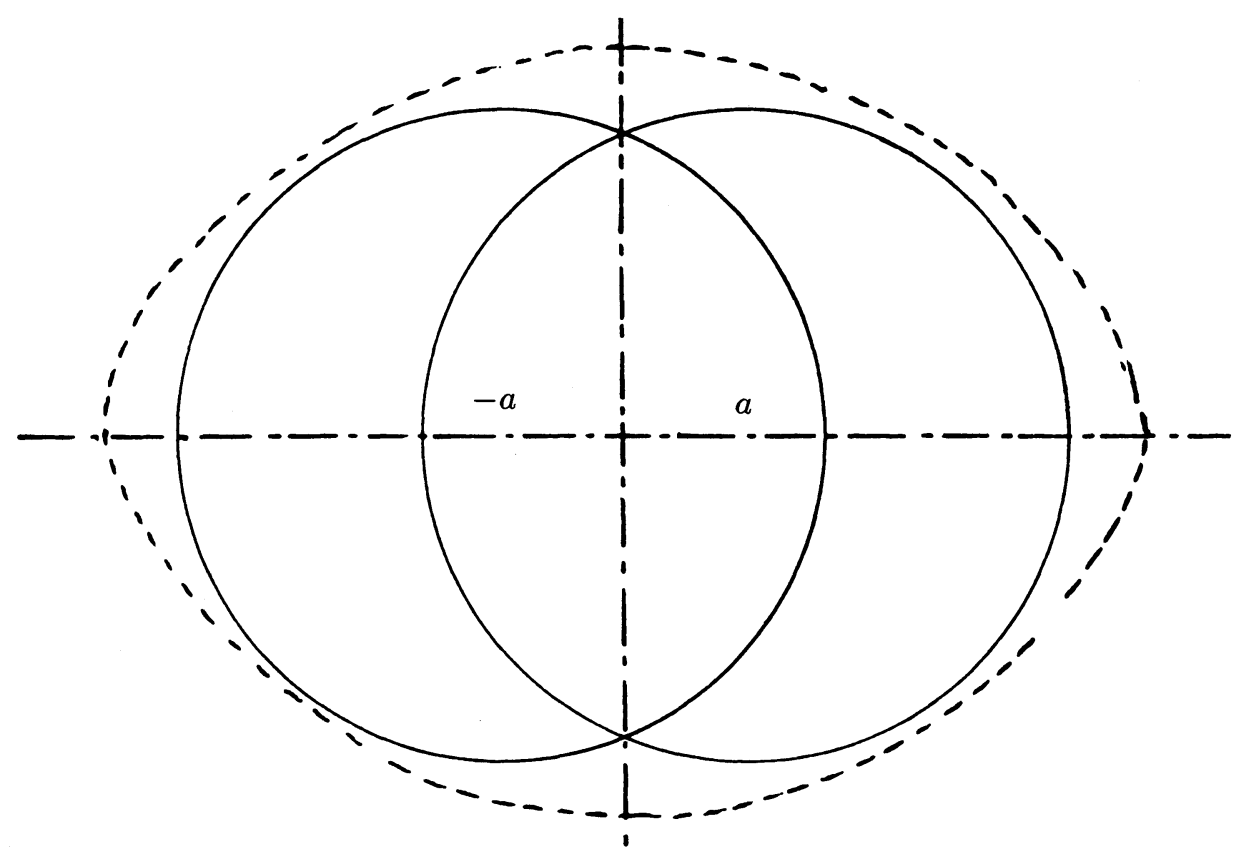

Tous ces corollaires reposent sur le calcul des ensembles $H_{\tau}$ définis cidessus; cela est laissé au lecteur. Signalons cependant que l'interaction cesse pour la raison suivante. Dans l'espace des fréquences, on a les situations suivantes :

si $t<\frac{a}{c_{2}} \frac{c_{1}}{\left(c_{1}^{2}-c_{2}^{2}\right)^{1 / 2}}$, il y a interaction :

si $t>\frac{a}{c_{2}} \frac{c_{1}}{\left(c_{1}^{2}-c_{2}^{2}\right)^{1 / 2}}$, il n'y a plus interaction :
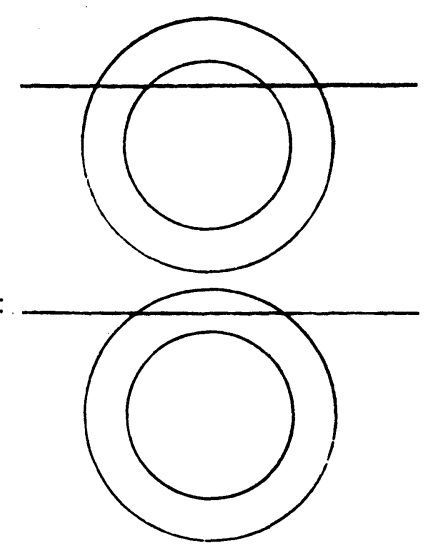

TOME $116-1988-\mathrm{N}^{\circ} 3$ 


\section{Calcul bilinéaire et paralinéarisation précisée}

Le but de cette partie est de construire un calcul symbolique bilinéaire, c'est-à-dire de définir les symboles, de les quantifier, et enfin de démontrer propriétés opératoires et formules symboliques. Ensuite, on démontrera les deux théorèmes de paralinéarisation précisée 2.4 et 2.5 .

Dans toute la suite $\rho$ désigne un réel positif non entier. Pour des raisons techniques qui apparaitront dans la suite, on est contraint de réduire la classe des opérateurs paradifférentiels.

DÉFINITION 2.1.

(i) $\underline{\Sigma}_{\rho}^{m}$ est l'ensemble des fonctions $a(x, \xi)$, somme pour $j$ variant de $0 \grave{a}[\rho]$, de fonctions $a_{j}(x, \xi)$, homogènes de degré $m-j$ en $\xi$ pour $|\xi| \geq 1$, et telles que

$$
\left|\partial_{\xi}^{\alpha} a_{j}(\cdot, \xi)\right|_{\rho-j+n / 2} \leq C_{j, \alpha}\langle\xi\rangle^{m-j-|\alpha|} .
$$

(ii) $\underline{\Sigma}_{\rho}^{m}(\Omega)=\left\{a(x, \xi) \mid \forall \varphi \in \mathcal{C}_{0}^{\infty}(\Omega) \quad \varphi a \in \underline{\Sigma}_{\rho}^{m}\right\}$.

(iii) Op $\underline{\Sigma}_{\rho}^{m}(\Omega)$ est l'ensemble des opérateurs de Op $\Sigma_{\rho}^{m}(\Omega)$ dont le symbole est dans $\underline{\Sigma}_{\rho}^{m}(\Omega)$.

Remarquons qu'il est clair que $\underline{\Sigma}_{\rho}^{m}(\Omega) \subset \Sigma_{\rho}^{m}(\Omega)$ et que Op $\underline{\Sigma}_{\rho}^{m}(\Omega) \subset$ $\mathrm{Op} \Sigma_{\rho}^{m}(\Omega)$.

Définition 2.2. - On appelle $\Sigma_{\rho}^{m, m^{\prime}}$ l'ensemble des fonctions $a(x, \xi, \eta)$ somme, pour $j$ variant de 0 à $[\rho]$, de fonctions $a_{j}(x, \xi, \eta)$ telles que :

(i) $\left|\partial_{\xi, \eta}^{\alpha} a_{j}(\cdot, \xi, \eta)\right|_{\rho+n / 2-j} \leq C_{j, \alpha}\langle\xi\rangle^{m}\langle\xi+\eta\rangle^{m^{\prime}-j-|\alpha|}$

(ii) Il existe deux réels $C$ et $C_{1}$, satisfaisant $0<C<C_{1}$ et tels que :

$$
\operatorname{Supp}_{\xi, \eta} a_{j} \subset\left\{(\xi, \eta): C|\xi| \leq|\eta| \leq C_{1}|\eta|\right\} .
$$

Définition 2.3. - Soient $T$ un paraproduit sur $\mathbb{R}^{n}$ et $a \in \Sigma_{\rho}^{m, m^{\prime}}$. On définit $T_{a}(u, v)$, pour $u$ et $v$ dans $\mathcal{S}$, par :

$$
\begin{aligned}
\mathcal{F}\left(T_{a}(u, v)\right)(\xi)=\frac{1}{(2 \pi)^{2 n}} \iint & T(\zeta-\xi-\eta, \xi+\eta) \\
& \times \hat{a}(\zeta-\xi-\eta, \xi, \eta) \hat{u}(\xi) \hat{v}(\eta) d \xi d \eta
\end{aligned}
$$

où $\mathcal{F}$ et ^ désignent ici, ainsi que dans toute cette partie la transformée de Fourier en la variable $x$.

Le premier travail à effectuer est de décomposer les symboles en séries rapidement convergentes de symboles plus simples.

Soit $a \in \Sigma_{\rho}^{m, m^{\prime}}$; on note encore par a l'une des fonctions $a_{j}$ de la DÉfinition 2.2. Posons $\tilde{a}\left(x, \theta_{1}, \theta_{2}\right)=a\left(x, \theta_{2}-\theta_{1}, \theta_{2}\right) \cdot\left\langle\theta_{2}\right\rangle^{-m^{\prime}}$. Soit $M$ un 
entier tel que $M>n$ et soit $\varphi \in \mathcal{C}_{0}^{\infty}(C)$ réalisant une partition de l'unité dyadique. Si l'on pose, pour $k \in \mathbb{Z}^{n}$ et $q \in \mathbb{N}$,

$$
a_{k, q}(x, \eta)=\int\left(\operatorname{Id}-\Delta_{\theta}\right)^{M} \tilde{a}\left(x, \eta, 2^{q} \theta\right) e^{-i(k \mid \theta)} \varphi(\theta) d \theta
$$

on a, d'après [11, pp. 46-48], l'existence d'une boule $B$ de $\mathbb{R}^{n}$ telle que :

$$
\begin{aligned}
a(x, \xi, \eta)=\sum_{k, q} \frac{\langle\xi+\eta\rangle^{m^{\prime}}}{\langle k\rangle^{M}} a_{k, q}(x, \eta) e^{i(k \mid \xi+\eta)} \varphi\left(2^{-q}(\xi+\eta)\right) & +a_{-1}(x, \xi, \eta)
\end{aligned}
$$

avec $\operatorname{Supp}_{\xi, \eta}\left(a_{-1}(x, \xi, \eta)\right) \subset\{(\xi, \eta) \mid \xi+\eta \in B\}$ et

$$
\left|a_{-1}(\cdot, \xi, \eta)\right|_{\rho+n / 2} \leq C\langle\eta\rangle^{m}
$$

Or, $a_{-1}$ est négligeable; en effet, on a :

Proposition 2.1. - Pour tout $(s, t)$ tel que $s+t \geq m, T_{a_{-1}}$ envoie $H^{s} \times H^{t}$ dans $H^{\infty}$.

Démonstration. - Il est clair que $\operatorname{Supp} \mathcal{F}\left(T_{a_{-1}}(u, v)\right) \subset B$; il suffit donc de démontrer que $T_{a_{-1}}$ envoie $H^{s} \times H^{t}$ dans $L^{2}$. Soit $m \in L^{2}$; on a alors :

$$
\begin{aligned}
I & =\int m(\zeta) \mathcal{F}\left(T_{a_{-1}}(u, v)\right)(\zeta) d \zeta \\
& =\int\left[\int \frac{m(\zeta+\xi+\eta) \hat{a}_{-1}(\zeta, \xi, \eta)}{\langle\xi\rangle^{s}\langle\eta\rangle^{t}} d \zeta\right] \Psi(\xi+\eta) f_{s}(\xi) g_{r}(\eta) d \xi d \eta
\end{aligned}
$$

avec $\Psi \in \mathcal{C}_{0}^{\infty}, f_{s}(\xi)=\langle\xi\rangle^{s} \hat{u}(\xi)$ et $g_{r}(\eta)=\langle\eta\rangle^{r} \hat{u}(\eta)$. D'où

$$
|I| \leq|m|_{0} \operatorname{Sup}_{\xi, \eta}\left(\frac{\left|a_{-1}(\cdot, \xi, \eta)\right|_{0}}{\langle\xi, \eta\rangle^{m}}\right)\left\|f_{s} * g_{r}\right\|_{0}
$$

d'où le résultat.

Dans la suite, on se ramène donc à une série rapidement convergente de symboles réduits du type :

$$
a_{q}(x, \eta) \varphi\left(2^{-q}(\xi+\eta)\right) \cdot \chi(\xi, \eta)
$$

avec $\left\|\varphi_{q}^{(\alpha)}\right\|_{0} \leq 2^{q\left(m^{\prime}-|\alpha|\right)}, \operatorname{Supp} \varphi_{q} \subset 2^{-q} C=C_{q}$, Supp $\chi \subset\{(\xi, \eta):$ $\left.C|\xi| \leq|\eta| \leq C_{1}|\xi|\right\}$ et $\left|D_{\eta}^{\alpha} a_{q}(\cdot, \eta)\right|_{\rho+n / 2} \leq C 2^{-q|\alpha|}\langle\eta\rangle^{m}$.

$$
\text { TOME } 116-1988-\mathrm{N}^{\circ} 3
$$


THÉORÈme 2.1. - Soient $a \in \Sigma_{\rho}^{m, m^{\prime}}, T$ et $T^{\prime}$ deux paraproduits sur $\mathbb{R}^{n}$; pour tout $(s, t)$ tel que $s+t>m+\frac{1}{2} n($ resp. $s+t \geq m)$. Alors : $T_{a}$ envoie $H^{s} \times H^{t}$ dans $H^{\sigma}\left(\right.$ resp. $\left.H_{-}^{\sigma}\right)$ avec $\sigma=s+t-m-m^{\prime}-\frac{1}{2} n$ et $T_{a}-T_{a}^{\prime}$ envoie $H^{s} \times H^{t}$ dans $H^{\sigma}\left(\right.$ resp. $\left.H_{-}^{\sigma}\right)$ avec $\sigma=s+t-m-m^{\prime}-\frac{1}{2} n+\rho$.

Démonstration. - Pour démontrer ce théorème, on a besoin du lemme suivant, qui sera d'ailleurs utilisé constamment dans la construction du calcul :

Lemme 2.1. - Soient $f$ et $g$ deux suites de $L^{2}\left(\mathbb{R}^{n}\right)$ telles qu'il existe une couronne $K$ telle que pour tout $r$, Supp $f_{r}$ et $\operatorname{Supp} g_{r}$ soient inclus dans $2^{r} K$; de plus $f_{r}$ et $g_{r}$ sont positives. On pose $|f|_{s}^{2}=\sum_{r} 2^{2 r s}\left|f_{r}\right|_{0}^{2}$. Soit $h_{q, r}(\theta, \eta)$ une suite de fonctions mesurables positives sur $\mathbb{R}^{n}$. Posons $N_{\mu}\left(h_{q}\right)=\operatorname{Sup}_{r}\left\{2^{-r \mu} \operatorname{Sup}_{\eta \in K_{r}}\left\|h_{q}(\cdot, \eta)\right\|_{L^{1}}\right\}$. Soit $\Psi_{q}$ une suite de fonctions positives supportées dans $2^{q} K$ tel que $\left\|\Psi_{q}\right\|_{L^{\infty}} \leq C 2^{-q \mu^{\prime}}$. Soit

$$
A_{q}(\zeta)=\sum_{r \geq q+N} \int h_{q, r}(\zeta-\xi-\eta, \eta) \Psi_{q}(\xi+\eta) f_{r}(\xi) g_{r}(\eta) d \xi d \eta
$$

Sous ces conditions, on a, pour tout $(s, t)$ tel que $s+t>\mu+\frac{1}{2} n$ (resp. $s+t \geq \mu$ )

$$
\left|A_{q}\right|_{0} \leq c_{q} 2^{-q\left(s+t-\mu-\mu^{\prime}-n / 2\right)} N_{\mu}\left(h_{q}\right)|f|_{s}|g|_{t}
$$

avec $c_{q} \in \ell^{2}\left(\right.$ resp. $\left.\ell^{\infty}\right)$.

Démonstration du lemme. - Etudions la quantité suivante :

$$
H_{q, r}=\iiint m(\zeta) h_{q, r}(\zeta-\theta, \eta) \Psi_{q}(\theta) f_{r}(\theta-\eta) g_{r}(\eta) d \theta d \eta d \zeta .
$$

D'après l'inégalité de Schwarz avec le poids $h_{q, r}(\zeta-\theta, \eta)$ on a

$$
\left[\int m(\zeta) h_{q}(\zeta-\theta, \eta) d \zeta\right]^{\frac{1}{2}} \leq N_{\mu}\left(h_{q}\right) 2^{r \mu / 2}\left[\int m^{2}(\zeta) h_{q}(\zeta-\theta, \eta) d \zeta\right]^{\frac{1}{2}} .
$$

On applique maintenant l'inégalité de Schwarz avec la mesure $d \theta$, d'où :

$$
H_{q, r} \leq\left(N_{\mu}\left(h_{q}\right)\right)^{\frac{1}{2}} 2^{r \mu / 2} \int g_{r}(\eta) a_{r, q}(\eta) b_{q, r}(\eta) d \eta
$$

avec

$$
\begin{aligned}
a_{r, q}(\eta) & =\left[\int \Psi_{q}(\theta) f_{r}^{2}(\theta-\eta) d \theta\right]^{1 / 2}, \\
b_{r, q}(\eta) & =\left[\iint m^{2}(\zeta) h_{q, r}(\zeta-\theta, \eta) d \theta d \zeta\right]^{1 / 2} .
\end{aligned}
$$

BULLETIN DE LA SOCIÉTÉ MATHÉMATIQUE DE FRANCE 
On a déjà la majoration $\left\|b_{r, q}\right\|_{0} \leq|m|_{0} N_{\mu}\left(h_{q}\right)^{1 / 2} 2^{r \mu / 2}$.

Si $s+t \geq \mu$, on a $\left\|a_{r, q}\right\|_{L^{1}} \leq 2^{q\left(n / 2+\mu^{\prime}\right)}\left|f_{r}\right|_{0}$ d'où

$$
\|\left. A_{q}\right|_{0} \leq 2^{q\left(\frac{n}{2}+\mu^{\prime}\right)} N_{\mu}\left(h_{q}\right)|f|_{s}|g|_{t} \sum_{r \geq q+N} c_{r} d_{r} 2^{-(s+t-\mu) r}
$$
d'où

Si $s+t>\mu+n / 2$, posons $c_{q, r}=2^{-2 q \mu^{\prime}} 2^{2 r s}\left\|a_{r, q}\right\|_{0}$. On a $\sum_{q, r} c_{q, r}^{2} \leq C$,

$$
\begin{aligned}
\left|A_{q}\right|_{0} & \leq N_{\mu}\left(h_{q}\right) 2^{q \mu^{\prime}} \sum_{r \geq q+N} 2^{-r\left(s+t-\frac{n}{2}-\mu\right)} c_{q, r}|f|_{s}|g|_{t} \\
& \leq c_{q} N_{\mu}\left(h_{q}\right) 2^{-q\left(s+t-\frac{n}{2}-\mu-\mu^{\prime}\right)}|f|_{s}|g|_{t}
\end{aligned}
$$

avec $c_{q}=\left(\sum_{r} c_{q, r}^{2}\right)^{1 / 2}$.

Revenons à la démonstration du THÉORÈme 2.1 :

$$
\mathcal{F}\left(T_{q}(u, v)\right)(\zeta)=\frac{1}{(2 \pi)^{2 n}} \sum_{\substack{r \geq q+N \\|\nu| \leq N_{0}}} \iint T(\zeta-\xi-\eta, \xi+\eta) \hat{a}_{q}(\zeta-\xi-\eta, \eta)
$$

On a clairement $\operatorname{Sp}\left(T_{q}(u, v)\right) \subset C_{q}^{\prime}$ couronne d'ordre $2^{+q}$. Or, en posant $\tilde{v}_{r}=\sum_{|\nu| \leq N_{0}}\left|\tilde{v}_{r-\nu}\right|$, on a

$$
\begin{aligned}
\left|\mathcal{F}\left(T_{q}(u, v)\right)(\zeta)\right| \leq C \sum_{r \geq q+N} \int\left|\hat{a}_{q}(\zeta-\xi-\eta, \eta)\right|\left|\varphi_{q}(\xi+\eta)\right| \\
\times\left|\hat{u}_{r}(\xi)\right| \tilde{v}_{r}(\eta) d \xi d \eta
\end{aligned}
$$

d'où le résultat d'opérance en appliquant le LEMME 2.1 avec

$$
h_{q, r}(\theta, \eta)=\hat{a}_{q}(\theta, \eta), \quad \Psi_{q}=\left|\varphi_{q}\right|, \quad f_{r}=\left|\hat{u}_{r}\right| \quad \text { et } \quad g_{r}=\tilde{v}_{r} .
$$

Pour l'invariance par changement de paraproduit, on étudie

$$
\begin{aligned}
\Delta_{a}(\zeta)=\frac{1}{(2 \pi)^{2 n}} \sum_{\substack{r \geq q+N \\
|\nu| \leq N_{0}}} \iint\left(T-T^{\prime}\right) & (\zeta-\xi-\eta, \xi+\eta) \hat{a}_{q}(\zeta-\xi-\eta, \eta) \\
& \times \varphi_{q}(\xi+\eta) \hat{u}_{r}(\xi) \hat{v}_{r-\nu}(\eta) d \xi d \eta .
\end{aligned}
$$

Vu que Supp $\Delta_{q} \subset C_{q}^{\prime}$, il suffit de démontrer que l'on a :

$$
\left|\Delta_{q}\right|_{0} \leq c_{q} 2^{-q\left(s+t-n / 2-m-m^{\prime}+\rho\right)}
$$

avec $\sum c_{q}^{2}<+\infty$

TOME $116-1988-\mathrm{N}^{\circ} 3$ 
On découpe spectralement $a_{q}$; vu le support de $T-T^{\prime}$, on a :

$$
\left|\Delta_{q}(\zeta)\right| \leq \sum_{r \geq q+N} \iint h_{q}(\zeta-\xi-\eta, \eta) \Psi_{q}(\xi+\eta) f_{r}(\xi) g_{r}(\eta) d \xi d \eta
$$

avec $h_{q}(\theta, \eta)=\sum_{|\nu| \leq N} \mid \varphi\left(2^{-q-\nu}(\theta) \hat{a}_{q}(\theta, \eta)\left|, \quad \Psi_{q}=\right| \varphi_{q}\left|, f_{r}=\right| \hat{u}_{r} \mid\right.$ et $g_{r}=\tilde{v}_{r}$; le résultat découle alors de l'application du Lemme 2.1.

Définition 2.3. - Soient $a \in \Sigma_{\rho}^{m, m^{\prime}}, b \in \underline{\Sigma}_{\rho}^{m_{1}}$ et $c \in \underline{\Sigma}_{\rho}^{m_{2}}$. Alors

$$
b \# a=\left.\sum_{|\alpha|+i+j \leq[\rho]} \frac{1}{\alpha !} \partial_{\xi}^{\alpha} b_{i}(x, \xi)\right|_{\xi+\eta} D_{x}^{\alpha} a_{j}(x, \xi, \eta),
$$

(ii) $a \#(b, c)=\sum_{|a|+|\beta|+i+j+\ell \leq[p]} \frac{1}{\alpha ! \beta !} \partial_{\xi}^{\alpha} \partial_{\eta}^{\beta} a_{i}(x, \xi, \eta) D_{x}^{\alpha} b_{j}(x, \xi) D_{x}^{\beta} c_{\ell}(x, \eta)$.

Remarquons qu'il résulte clairement du caractère d'algèbre de $H^{s}$ pour $s>\frac{1}{2} n$ que l'on a $b \# a \in \Sigma_{\rho}^{m, m^{\prime}+m_{1}}$ et $a \#(b, c) \in \Sigma_{\rho}^{m+m_{1}+m_{2}, m^{\prime}}$.

On va maintenant énoncer le théorème fondamental du calcul symbolique.

THÉORÈME 2.2. - Soient $a \in \Sigma_{\rho}^{m, m^{\prime}}, b \in \mathrm{Op} \underline{\Sigma}_{\rho}^{m_{1}}$ et $c \in \underline{\Sigma}_{\rho}^{m_{2}}$. On a :

(i) pour tout $(s, t)$ tel que $s+t>m+\frac{1}{2} n$ (resp. $\left.s+t \geq m\right)$, l'application $T_{b} \circ T_{a}-T_{b \# a}$ envoie $H^{s} \times H^{t}$ dans $H^{\sigma}$ (resp. $H_{-}^{\sigma}$ ) avec $\sigma=s+t-\frac{1}{2} n-m-m^{\prime}-m_{1}+\rho$,

(ii) pour tout $(s, t)$ tel que $s+t \geq m+m_{1}+m_{2}$, l'application $T_{a} \circ\left(T_{b} \otimes T_{c}\right)-T_{a \#(b, c)}$ envoie $H^{s} \times H^{t}$ dans $H^{\sigma}$ (resp. $\left.H_{-}^{\sigma}\right)$ avec $\sigma=s+t-\frac{1}{2} n-m-m_{1}-m_{2}-m^{\prime}+\rho$.

La démonstration de ce théorème, assez longue et technique, est effectuée en appendice.

On va maintenant localiser de manière standard le calcul global obtenu.

Définition 2.4. - Soit $\Omega$ un ouvert de $\mathbb{R}^{n}$.

(i) On désigne par $\Sigma_{\rho}^{m, m^{\prime}}(\Omega)$ l'ensemble des fonctions $a(x, \xi, \eta)$ telles que, pour toute $\varphi$ dans $\mathcal{C}_{0}^{\infty}(\Omega)$ on ait $\varphi a \in \Sigma_{\rho}^{m, m^{\prime}}$.

(ii) Soient $a \in \Sigma_{\rho}^{m, m^{\prime}}(\Omega), b \in \Sigma_{\rho}^{m_{1}}(\Omega)$ et $c \in \Sigma_{\rho}^{m_{2}}(\Omega)$ :

$$
\begin{aligned}
b \# a(x, \xi, \eta) & =\left.\sum_{|\alpha|+i+j \leq[\rho]} \frac{1}{\alpha !} \partial_{\xi}^{\alpha} b_{i}(x, \xi)\right|_{\xi+\eta} D_{x}^{\alpha} a_{j}(x, \xi, \eta), \\
a \#(b, c)(x, \xi, \eta) & =\sum_{|\alpha|+|\beta|+i+j+\ell \leq[\rho]} \frac{1}{\alpha ! \beta !} \partial_{\xi}^{\alpha} \partial_{\eta}^{\beta} a_{i}(x, \xi, \eta) \\
& \times D_{x}^{\alpha} b_{j}(x, \xi) D_{x}^{\beta} c_{\ell}(x, \eta) .
\end{aligned}
$$

BULLETIN DE LA SOCIÉTÉ MATHÉMATIQUE DE FRANCE 
Il est clair que $b \# a \in \Sigma_{\rho}^{m, m^{\prime}+m_{1}}(\Omega)$ et que $a \#(b, c)$ appartient à $\Sigma_{\rho}^{m+m_{1}+m_{2}, m^{\prime}}(\Omega)$.

Définition 2.5. - Soit $\Omega$ un ouvert de $\mathbb{R}^{n}$; on dit que $A \in \mathrm{Op} \Sigma_{\rho}^{m, m^{\prime}}(\Omega)$ si et seulement si les trois conditions suivantes sont vérifiées pour tout $(s, t)$ tel que $s+t>m+\frac{1}{2} n$ (resp. $s+t \geq m$ ) :

(i) $A$ envoie $H_{\text {loc }}^{s}(\Omega) \times H_{\text {loc }}^{t}(\Omega)$ dans $\mathcal{D}^{\prime}(\Omega)$;

(ii) $A$ est à support propre au sens suivant : pour tout compact $K \operatorname{de} \Omega$, il en existe un $K^{\prime}$ tel que : $\operatorname{si} \operatorname{Supp}(u \otimes v) \subset K \times K$, alors $\operatorname{Supp} A(u, v) \subset K^{\prime}$, et si $\operatorname{Supp}(u \otimes v) \subset \complement K^{\prime} \times K^{\prime}$, alors $\operatorname{Supp} A(u, v) \subset \complement K$;

(iii) Il existe $a \in \Sigma_{\rho}^{m, m^{\prime}}(\Omega)$, tel que, pour tout compact $K$ de $\Omega$ et toute $\chi \in \mathcal{C}_{0}^{\infty}(\Omega)$ valant 1 au voisinage de $K$, l'application $A-\chi T_{\chi a}$ envoie $H_{K}^{s} \times H_{K}^{t}$ dans $H^{\sigma}$ (resp. $H_{-}^{\sigma}$ ) avec $\sigma=s+t-\frac{1}{2} n-m-m^{\prime}+\rho$. de $A$.

Définition 2.6. - Lorsque (iii) est réalisé, on dit que $a$ est un symbole

Le théorème suivant permettra une utilisation locale de ce calcul.

THÉORÈME 2.3. - Soit $\Omega$ un ouvert de $\mathbb{R}^{n}$.

(i) Pour tout a dans $\Sigma_{\rho}^{m, m^{\prime}}(\Omega)$, il existe $A$ dans $O p \Sigma_{\rho}^{m, m^{\prime}}(\Omega)$ tel que a soit un symbole de $A$.

(ii) Soient a un symbole de $A \in \mathrm{Op} \Sigma_{\rho}^{m, m^{\prime}}(\Omega)$ et $b$ (resp. c) un symbole de $B \in \mathrm{Op} \underline{\Sigma}_{\rho}^{m_{1}}(\Omega)$ (resp. $C \in \mathrm{Op} \underline{\Sigma}_{\rho}^{m_{2}}(\Omega)$ ). Alors $B \circ A \in$ $\mathrm{Op} \Sigma_{\rho}^{m, m^{\prime}+m_{1}}(\Omega)$ et b\#a est un symbole de $B \circ A ; A \circ(B \otimes C) \in$ $\mathrm{Op} \Sigma_{\rho}^{m_{1}+m_{1}+m_{2}, m^{\prime}}(\Omega)$ et $a \#(b, c)$ est un symbole de $A \circ(B \otimes C)$.

Démonstration. - Soit $\left(w_{j}, \varphi_{j}\right)_{j \in \mathbb{N}}$ une partition de l'unité localement finie de $\Omega$, soit $\chi_{j} \in \mathcal{C}_{0}^{\infty}\left(w_{j}\right)$ valant 1 au voisinage de $\operatorname{Supp} \varphi_{j}$. On pose

$$
A(u, v)=\sum_{i, j} \chi_{i} \chi_{j} T_{\chi_{i} \chi_{j} a}\left(\varphi_{i} u, \varphi_{j} v\right)
$$

Vérifier les points (i) et (ii) de la DÉfinition 2.5 est facile. Pour montrer que $a$ est un symbole de $A$, il suffit d'appliquer le THÉORÈme 2.2 en suivant la démarche de [6] lors du passage du calcul global au calcul local. Il en est de même pour le point (ii).

Il faut maintenant préciser la définition des opérateurs paradifférentiels en vue des ThÉORÈmes 2.4 et 2.5 .

Définition 2.7.

(i) Soit $\Omega$ un ouvert de $\mathbb{R}^{n}$; on appelle $\mathcal{P}_{\rho}^{m}(\Omega)$ l'ensemble des éléments de $\Sigma_{\rho}^{m}(\Omega)$ polynomiaux en $\xi$.

$$
\text { TOME } 116-1988-\mathrm{N}^{\circ} 3
$$


(ii) Soient $T$ un paraproduit sur $\mathbb{R}^{n},\left(\varphi_{j}, w_{j}\right)_{j \in \mathbb{N}}$ une partition de l'unité $\mathcal{C}^{\infty}$ de $\Omega,\left(\chi_{j}\right)_{j \in \mathbb{N}}$ une suite de fonctions $\mathcal{C}_{0}^{\infty}\left(w_{j}\right)$ telle que $\chi_{j}$ vaille 1 au voisinage $\operatorname{de} \operatorname{Supp} \varphi_{j}$ et $a \in \mathcal{P}_{\rho}^{m}(\Omega)$; on pose :

$$
\operatorname{OP} P_{T}(a) u=\sum_{j \in \mathbb{N}} \chi_{j} T_{\chi_{j} a} \varphi_{j} u
$$

Il est clair que OP $P_{T}(a)$ opère proprement et continûment de $\mathcal{D}^{\prime}(\Omega)$ dans $\mathcal{D}^{\prime}(\Omega)$. L'intérêt de cette définition apparait dans la proposition suivante.

Proposition 2.2.

(i) Pour tout compact $K$ de $\Omega$ et toute $\chi \in \mathcal{C}_{0}^{\infty}(\Omega)$ valant 1 au voisinage de $K$, OP $P_{T}(a)-\chi T_{\chi a}$ envoie les distributions à support dans $K$ dans $\mathcal{C}_{0}^{\infty}$ et ce pour tout $a \in \mathcal{P}_{\rho}^{m}(\Omega)$; en particulier OP $P_{T}(a) \in \mathrm{Op} \Sigma_{\rho}^{m}(\Omega)$ et $\mathrm{OP} P_{T}(a)$ est indépendant de la partition de l'unité choisie modulo un opérateur infiniment régularisant.

(ii) OP $P_{T}(a)$ est aussi microlocal que l'on veut i.e. pour tout $\epsilon>0$, il existe $\epsilon_{0}>0$ tel que, si $\operatorname{Supp} T \subset\left\{(\xi, \eta):|\xi| \leq \epsilon_{0}|\eta|\right\}$ alors $W F\left(\mathrm{OP} P_{T}(a) \cdot u\right) \subset\left\{(x, \xi) \in T^{*} \Omega \mid d\left(\xi /|\xi|, W F(u) \cap S^{n-1}\right) \leq \epsilon\right\}$.

Pour la démonstration, voir [10].

Nous pouvons maintenant énoncer les deux théorèmes de paralinéarisation précisée :

ThÉORÈme 2.4. - Soient $\Omega$ un ouvert de $\mathbb{R}^{n}, u \in H_{\mathrm{loc}}^{s}(\Omega)$ avec $s>n / 2, f \mathcal{C}^{\infty}$ au voisinage des valeurs de $u$. Pour tout paraproduit $T$ sur $\mathbb{R}^{n}$, il existe $R \in \mathrm{Op} \Sigma_{s-n / 2}^{0,0}(\Omega)$ tel que

$$
f(u)-\mathrm{OP} P_{T}\left(f^{\prime}(u)\right) \cdot u-R(u, u) \in H_{\mathrm{loc}}^{3 s-n}(\Omega) .
$$

THÉorÈme 2.5. - Soient $\Omega$ un ouvert de $\mathbb{R}^{n}$ et u une solution $H_{\mathrm{loc}}^{s}(\Omega)$ de l'équation

$$
f\left(x, u, \partial^{\beta} u\right)_{|\beta| \leq m}=0
$$

avec $s>n / 2+m+1-d(d$ valant 2 dans le cas semi-linéaire, 1 dans le cas quasi-linéaire, 0 sinon). Pour tout paraproduit $T$ sur $\mathbb{R}^{n}$, il existe $R \in$ $\mathrm{Op} \Sigma_{s-n / 2-m+d-1}^{2 m-d, 0}(\Omega)$ tel que, si $p(x, \xi)=\sum_{|\alpha| \leq m} \frac{\partial f}{\partial u_{\alpha}}\left(x, u, \partial^{\beta} u\right)(i \xi)^{\alpha}$, on ait :

$$
\mathrm{OP} P_{T}(p) \cdot u+R(u, u) \in H_{\mathrm{loc}}^{3 s-n-3 m+2 d-1}(\Omega) \text {. }
$$




\section{Remarques.}

Il n'y a aucune hypothèse sur la nature de l'équation.

Le ThÉORÈmE 2.5 est très important; c'est lui qui transforme une équation non linéaire en une équation paradifférentielle bilinéaire.

Nous démontrerons uniquement le THÉORÈmE 2.4, la démonstration du ThÉorème 2.5, strictement analogue dans son principe à celle du THÉORÈme 2.4, étant d'écriture lourde est omise.

Démonstration. - On convient, dans cette démonstration, que toutes les égalités sont écrites modulo une fonction $H^{3 s-n}$.

D'après la Proposition 2.2 et le calcul paradifférentiel bilinéaire il suffit de démontrer un théorème global. On utilise le théorème d'approximation 1.3 .1 de [10] à l'ordre 2 ; d'après ce théorème, on a $f(u)=$ $\sum_{i=1}^{3} \Delta^{(i)}(u)$ avec

$$
\begin{aligned}
& \Delta^{(1)}(u)=\sum_{q \geq 0} S_{q+N_{2}}\left(f^{\prime}(u)\right) \cdot u_{q} \\
& \Delta^{(2)}(u)=-\sum_{q \geq 0} u_{q} S_{q+N_{2}}\left(\sum_{p \geq q} u_{p} S_{p+N_{1}}\left(f^{\prime}(u)\right)\right), \\
& \Delta^{(3)}(u)=\sum_{q \geq 0} u_{q}^{2} S_{q+N_{2}}\left(f^{\prime \prime}(u)\right) .
\end{aligned}
$$

On a aussi $\Delta^{(1)}(u)=T_{f^{\prime}(u)} \cdot u+R\left(f^{\prime}(u), u\right)$ avec

$$
R\left(v, v^{\prime}\right)=\sum_{q \geq 0}\left(S_{q+N_{2}}(v)-S_{q-N_{0}}(v)\right) \cdot v_{q}^{\prime} .
$$

Or $f^{\prime}(u)=T_{f^{\prime \prime}(u)} \cdot u+\tilde{\rho}$ avec $\tilde{\rho} \in H^{2 s-n / 2}$ donc

$$
\Delta^{(1)}(u)=T_{f^{\prime}(u)} \cdot u+R\left(T_{f^{\prime \prime}(u)} \cdot u, u\right) .
$$

Il est facile de voir que l'on a :

$$
\Delta^{(2)}(u)=-\sum_{q} u_{q} \theta\left(2^{-q} D\right)\left(\sum_{|p-q| \leq N} u_{p} S_{p-N_{0}}\left(f^{\prime}(u)\right)\right) .
$$

On met clairement les $\Delta^{(i)}(u)$ sous la forme d'opérateurs paradifférentiels de reste globaux grâce aux deux lemmes suivants :

LEMme 2.2. - Soient $\theta$ et $\tilde{\theta}$ deux fonctions de $\mathcal{C}_{0}^{\infty}(C), C$ étant une couronne de $\mathbb{R}^{n}$; posons

$$
\begin{aligned}
R_{3}(a, u, v) & =\sum_{q} 2^{-q k} \theta\left(2^{-q} D\right) u \tilde{\theta}\left(2^{-q} D\right) v \cdot S_{q-N_{0}}(a) \\
R_{2}(u, v) & =\sum_{q} 2^{-q k} \theta\left(2^{-q} D\right) u \tilde{\theta}\left(2^{-q} D\right) v, \quad(k \in \mathbb{N}) .
\end{aligned}
$$

TOME $116-1988-\mathrm{N}^{\circ} 3$ 
Pour tout $r>k$ et tout $(s, t)$ tels que $s+t>n / 2-k$, on $a:$

$$
\left|R_{3}(a, u, v)-T_{a} R_{2}(u, v)\right|_{s+t-n / 2+r} \leq C\|a\|_{r-k}|u|_{s}|v|_{t}
$$

Démonstration. - Posons $u_{q}=\theta\left(2^{-q} D\right) u$ et $v_{q}=\tilde{\theta}\left(2^{-q} D\right) v$. Un calcul très simple montre que l'on a $R_{3}(a, u, v)=T_{a} R_{2}(u, v)+\sum_{i=1}^{2} R^{(i)}(a, u, v)$ avec

$$
\begin{aligned}
& \begin{array}{r}
R^{(1)}(a, u, v)=\sum_{\left|p-p_{1}\right| \leq N} \varphi\left(2^{-p} D\right)\left\{\left(S_{p-N_{0}}-S_{p_{1}-N_{0}}\right)(a)\right. \\
\left.\times \varphi\left(2^{-p_{1}} D\right) R_{2}(u, v)\right\}
\end{array} \\
& R^{(2)}(a, u, v)=\sum_{p \leq q+N^{\prime}} 2^{-q k} \varphi\left(2^{-p} D\right)\left\{\left(S_{q-N_{0}}(a)-S_{p-N_{0}}(a)\right) u_{q} v_{q}\right\} .
\end{aligned}
$$

Le terme $R^{(1)}$ est sans problème. Pour $R^{(2)}$, posons

$$
W_{q}=\sum_{p \leq q+N^{\prime}} \varphi\left(2^{-p} D\right)\left\{\left(S_{q-N_{0}}(a)-S_{p-N_{0}}(a)\right) 2^{-q k} u_{q} v_{q}\right\}
$$

Soit $\lambda$ tel que $-k<\lambda-r<s+t-n / 2-k$;

$$
\begin{aligned}
\left|\langle D\rangle^{\lambda} W_{q}\right|_{0} & \lesssim \sum_{p \leq q+N^{\prime}} 2^{p \lambda} \varphi\left(2^{-p} D\right)\left\{\left(S_{q-N_{0}}(a)-S_{p-N_{0}}(a)\right) 2^{-q k} u_{q} v_{q}\right\} \\
& \lesssim \sum_{p \leq q+N^{\prime}} 2^{p \lambda} 2^{-p(r-k)}\|a\|_{r-k} 2^{-q(s+t-n / 2+k)} \tilde{c}_{q} \\
& \lesssim \tilde{c}_{q} 2^{-q(s+t-n / 2+r-\lambda)}
\end{aligned}
$$

avec $\sum \tilde{c}_{q}^{2}<+\infty$, d'où le résultat.

Lemme 2.3. - Soient $\theta$ et $\tilde{\theta}$ deux fonctions $\mathcal{C}_{0}^{\infty}(C), C$ étant une couronne de $\mathbb{R}^{n}, u \in H^{s}$ et $a \in C^{r}$ avec $r>0$; on a alors, en posant $N=[r]$,

$$
\begin{aligned}
\mid \tilde{\theta}\left(2^{-q} D\right) & \left\{S_{q-N_{0}}(a) \theta\left(2^{-q} D\right) u\right\} \\
& -\left.\sum_{|a| \leq N} \frac{2^{-q|\alpha|}}{\alpha !} S_{q-N_{0}}\left(D^{\alpha} a\right) \theta \tilde{\theta}^{(\alpha)}\left(2^{-q} D\right) u\right|_{0} \leq c_{q} 2^{-q(s+r)}
\end{aligned}
$$

$\operatorname{avec} \sum c_{q}^{2} \leq C\|a\|_{r}^{2}|u|_{s}^{2}$ 
Démonstration. - C'est standard; on écrit une formule de Taylor à l'ordre $N+1$, et il vient, en désignant par $W_{q}$ la différence à majorer et par $h$ la transformée de Fourier inverse de $\tilde{\theta}$ :

$$
\begin{array}{r}
W_{q}(x)=\sum_{|\alpha|=N+1} \iint_{0}^{1} \frac{(1-t)^{N}}{N !} 2^{-q(N+1)} S_{q-N_{0}}\left(D^{\alpha} a\right)\left(x+(t-1) 2^{-q} y\right) \\
\times\left(\theta\left(2^{-q} D\right) u\right)(x-y) y^{\alpha} h(y) d y d t
\end{array}
$$

d'où $\left|W_{q}\right|_{0} \leq c_{q}\|a\|_{r}|u|_{s} 2^{-q(s+r)}$, avec $\sum c_{q}^{2} \leq C$.

\section{3. Étude de la régularité microlocale du reste; démonstration des théorèmes 11 et 1.2}

Après avoir démontré les ThÉORÈmes 2.4 et 2.5 , le problème est d'obtenir, lorsque $u$ est solution de (E), des propriétés microlocales sur $R(u, u)$. Cela nécessite la considération d'algèbres $H^{s}(P)(P$ désignant le linéarisé de $f$ pour $u$ ), destinées à contenir $u$. Elles permettent, par le biais du lemme de l'hyperplan tangent 3.3, qui dit qu'une dérivation elliptique en un point n'appartenant pas au plan tangent à Car $\left.p_{m}\right|_{x}$ se factorise à travers les opérateurs bilinéaires, de préciser l'interaction de deux singularités de directions caractéristiques antipodales.

Définition 3.1. - Soit $P \in \mathrm{Op} \Sigma_{\rho}^{m}(\Omega)$; on pose

$$
\begin{aligned}
H^{s}(P)=\left\{u \in H_{\mathrm{loc}}^{s}(\Omega) \mid P^{j} u \in H_{\mathrm{loc}}^{s-(m-1) j}(\Omega)\right. & \forall j \leq[\rho], \\
P^{j} u \in H^{s+\rho-m j}, & \text { si } j=[\rho]+1\} .
\end{aligned}
$$

Avant d'entamer l'étude de ces espaces, on donne le lemme de commutation suivant.

Lemme 3.1. - Soient $P \in \mathrm{Op} \Sigma_{\rho}^{m}(\Omega)$ et $A \in \mathrm{Op} \Sigma_{\rho}^{m^{\prime}}(\Omega)$. Pour tout entier $j$, on a la propriété $\left(C_{j}\right)$ suivante :

$\left(C_{j}\right) \quad\left\{\begin{array}{l}\text { il existe des opérateurs paradifférentiels } B_{i}, \\ \text { avec } 0 \leq i \leq \inf (j,[\rho])=N, \text { tels que } \\ P^{j} A=\sum_{i=0}^{N} B_{i} P^{j-i} \text { et } B_{i} \in \mathrm{Op} \Sigma_{\rho-i}^{i(m-1)+m^{\prime}}(\Omega) .\end{array}\right.$

Démonstration. - On procède par récurrence sur $j$. La propriété $\left(C_{1}\right)$ est clairement vraie. Soit $j<[\rho]$ et supposons $\left(C_{j}\right)$ vraie. On a alors

$$
\begin{aligned}
p^{j+1} A & =\sum_{i=0}^{j} P B_{i} P^{j-i} \\
& =\sum_{i=1}^{j+1}\left(B_{i}+\left[P, B_{i-1}\right]\right) P^{j+1-i}+B_{0} P^{j+1}+\left[P, B_{j}\right],
\end{aligned}
$$

TOME $116-1988-\mathrm{N}^{\circ} 3$ 
d'où $\left(C_{j+1}\right)$; d'où $\left(C_{[\rho]}\right)$ par récurrence.

Si $j \geq[\rho]$; supposons $\left(C_{j}\right)$; on a alors

$$
\begin{aligned}
P^{j+1} A & =\sum_{i=0}^{[\rho]} P B_{i} P^{j-1} \\
& =\sum_{i=0}^{[\rho]-1}\left(\left[B_{i-1}, P\right]+B_{i}\right) P^{j-i}+B_{[\rho]} P^{j+1-[\rho]}
\end{aligned}
$$

d'où $\left(C_{j+1}\right)$ et le lemme.

Corollaire 3.1. - Soient $P \in \mathrm{Op} \Sigma_{\rho}^{m}(\Omega)$ et $A \in \mathrm{Op} \Sigma_{\rho}^{m^{\prime}}(\Omega)$. Alors $A$ envoie $H^{s}(P)$ dans $H^{s-m^{\prime}}(P)$.

La proposition suivante justifie l'introduction de ces espaces qui, vu que $H^{s+\rho} \subset H^{s}(P)$, et vu le Corollaire 3.1 sont des algèbres.

Proposition 3.1. - Soit $u$ solution de $f\left(x, u, \partial^{\beta} u\right)_{|\beta| \leq m}=0$ sur $\Omega$ ouvert de $\mathbb{R}^{n}, u$ étant $H_{\mathrm{loc}}^{s}(\Omega)$ avec $s>\frac{1}{2} n+m+1-d$, alors :

$$
\text { si } \sigma\left(P_{m}\right)(x, \xi)=\sum_{|\alpha|=m} \frac{\partial f}{\partial u_{\alpha}}(i \xi)^{\alpha}, \quad \text { alors } \quad u \in H^{s}\left(P_{m}\right)
$$

Démonstration. - Posons $\rho=s-\frac{1}{2} n-m+d$. Considérons l'énoncé

$$
\text { pour tout } i \leq j, \quad P_{m}^{i} u \in H_{\mathrm{loc}}^{s-(m-1) i}(\Omega) \text {. }
$$

La formule de paralinéarisation standard assure $\left(\mathcal{A}_{1}\right)$. Cette formule, jointe au Lemme 3.1 assure que $\left(\mathcal{A}_{j}\right)$ entraîne $\left(\mathcal{A}_{j+1}\right)$ pour $j \leq[\rho]$; d'où le résultat.

On va maintenant microlocaliser $H^{s}\left(P_{m}\right)$ au voisinage d'un point $\left(x_{0}, \xi_{0}\right) \in \operatorname{Car} p_{m}$ et se ramener dans le même temps au cas de l'ordre 1 .

Quitte à faire un changement de coordonnées, on peut supposer que $\xi_{0}=(1,0, \ldots, 0)$. Soit $\chi \in \mathcal{C}^{\infty}\left(\mathbb{R}^{n}\right)$, homogène de degré 0 pour $|\xi| \geq 1$, supportée dans un petit voisinage conique de $\xi_{0}$, et nulle pour $|\xi| \leq \frac{1}{2}$; on définit alors $Q \in \mathrm{Op} \Sigma_{\rho}^{1}(\Omega)$ par

$$
\sigma(Q)(x, \xi)=(\chi(\xi)+\chi(-\xi)) \frac{p_{m}(x, \xi)}{\xi_{1}^{m-1}}
$$

Il est très facile de voir que l'on a : 
Lemme 3.2. $-H^{s}(P) \subset H^{s}(Q)$.

On peut maintenant énoncer le lemme de l'hyperplan tangent :

Lemme 3.3 (lemme de l'hyperplan tangent). - Soit $q_{1} \in \mathrm{Op} \underline{\Sigma}_{\rho}^{1}(\Omega)$ tel que, pour tout $(x, \xi) \in T^{*} \Omega$ et tout $\alpha \in \mathbb{R}$, on ait $q_{1}(x, \alpha \xi)=\alpha q_{1}(x, \xi)$. Soit $\left(x_{0}, \xi_{0}\right) \in T^{*} \Omega$ tel que :

$$
q_{1}\left(x_{0}, \xi_{0}\right)=0 \quad \text { et } \quad d_{\xi} q_{1}\left(x_{0}, \xi_{0}\right) \neq 0 .
$$

Soient $K$ et $K^{\prime}$ deux ouverts coniques de $\mathbb{R}^{n}$ tels que :

$$
\bar{K}^{\prime} \subset K \quad \text { et }\left.\quad \bar{K} \cap T_{\xi_{0}} \operatorname{Car} q_{1}\right|_{x_{0}}=\emptyset ;
$$

Il existe alors : un voisinage ouvert $\Omega_{0}$ de $x_{0}$, un voisinage conique $K_{0}$ de $\xi_{0}$, un symbole pseudodifférentiel $\lambda$ d'ordre 1 , supporté dans $\Omega_{0} \times K$, et elliptique sur $\Omega_{0} \times K^{\prime}$, tels que pour tout $a_{ \pm} \in \mathrm{Op} S^{0}\left(\Omega_{0}\right)$ tel que $S E\left(a_{ \pm}\right) \subset \Omega_{0} \times \pm K_{0}$, il existe $r \in \Sigma_{\rho}^{0,0}\left(\Omega_{0}\right)$ tel que $\operatorname{Supp} r \subset \Omega_{0} \times K_{0} \times$ $-K_{0}$ et

$$
\lambda(x, \xi+\eta) a_{+}(x, \xi) a_{-}(x, \eta)=r(x, \xi, \eta)\left(q_{1}(x, \xi)+q_{1}(x, \eta)\right) .
$$

\section{Remarques.}

(a) Un lemme analogue est énoncé dans [5] dans le cas où le symbole $q_{1}$ provient d'un opérateur différentiel d'ordre 2 strictement hyperbolique.

(b) Le lemme signifie qu'il existe une dérivation $\lambda$ elliptique sur $\Omega_{0} \times K^{\prime}$ telle que, modulo les localisations convenables, elle s'exprime au travers d'un opérateur de reste, par des dérivations en $q_{1}$ qui seront bien supportées par une fonction de $H^{s}\left(Q_{1}\right)$.

(c) Le terme de droite de l'égalité (3.1) est dans $\underline{\Sigma}_{\rho}^{1,0}\left(\Omega_{0}\right)$; par contre, le terme de gauche est dans $\Sigma_{\infty}^{0,1}\left(\Omega_{0}\right)$ (c'est-à-dire que l'on perd sur l'un des indices ce que l'on gagne sur l'autre). En effet, considérons la famille fondamentale de voisinages coniques de $\xi_{0}$, supposé de norme 1 , définie par $K^{(\delta)}=\left\{\xi \in \mathbb{R}^{n}|0 \leq| \xi\left|-\left(\xi \mid \xi_{0}\right) \leq \delta\right| \xi \mid\right\}$. Vu la localisation de $K$, il existe $C<1$ tel que, si $\zeta \in K$, alors $\left|\left(\zeta \mid \xi_{0}\right)\right| \leq C|\zeta|$. Il est alors facile de voir que l'on a, pour tout $(\xi, \eta)$ dans $K^{(\delta)} \times-K^{(\delta)}$ tel que $\xi+\eta \in K$

$$
\begin{gathered}
|\xi+\eta| \leq \frac{\delta}{1-C}(|\xi|+|\eta|), \\
C|\eta| \leq|\xi| \leq C_{1}|\eta| .
\end{gathered}
$$

dès que $\delta$ est assez petit.

$$
\text { TOME } 116-1988-\mathrm{N}^{\circ} 3
$$


Démonstration. - Quitte à faire un changement de coordonnées, on peut supposer que $\xi_{0}=(1,0, \ldots, 0)$ et que l'on a pour tout $i \in$ $\{1, \ldots, n-1\}$

$$
\partial_{\xi_{i}} q_{1}\left(x_{0}, \xi_{0}\right)=0 \quad \text { et } \quad \partial_{\xi_{n}} q_{1}\left(x_{0}, \xi_{0}\right)=1
$$

Posons $\widehat{K}=\left\{(\xi, \eta) \in K_{0} \times-K_{0} \mid \xi+\eta \in K\right\}$. D'après (3.2) et (3.3), on peut supposer, quitte à restreindre $K_{0}$ que, si $(\xi, \eta) \in \widetilde{K}$, alors $|\xi+\eta| \leq \frac{1}{2}|\xi|$. D'après $(3.4)$ on a $\xi_{n}=q_{1}(x, \xi)+s(x, \xi)$ avec $s\left(x_{0}, \xi_{0}\right)=0$ et $d_{\xi} s\left(x_{0}, \xi_{0}\right)=0$. D'autre part, vu que $s(x,-\xi)=s(x, \xi)$ on a

$$
s(x, \xi)+s(x, \eta)=\sum_{j=1}^{n} \int_{0}^{1}\left(\xi_{j}+\eta_{j}\right) \partial_{\xi_{j}} s(x,-\xi+t(\xi+\eta)) d t
$$

Soit $(\xi, \eta) \in \widetilde{K}$ et posons

$$
b(x, \xi, \eta)=\sum_{j=1}^{n} \frac{\xi_{j}+\eta_{j}}{\xi_{n}+\eta_{n}} \partial_{\xi_{j}} s(x,-\xi+t(\xi+\eta)) d t .
$$

La première étape consiste à démontrer que, quitte à restreindre $K_{0}$, on a, pour tout $(\xi, \eta) \in \widetilde{K},|b(x, \xi, \eta)| \leq \frac{1}{2}$. En effet, il existe $M>0$ tel que, si $(\xi, \eta) \in \widetilde{K}$, alors $\left|\left(\xi_{j}+\eta_{j}\right) /\left(\xi_{n}+\eta_{n}\right)\right| \leq M$. Vu que $\partial_{\xi_{j}} s(x, \theta)$ est homogène en $\theta$ pour $|\theta| \geq 1$ et que $\partial_{\xi_{j}} s\left(x_{0}, \xi_{0}\right)=0$, il existe $\Omega_{0}$ voisinage de $x_{0}$, un $K_{0}^{\prime}$ voisinage conique de $\xi_{0}$ tels que, pour tout $(x, \theta)$ dans $\Omega_{0} \times K_{0}^{\prime} \cup-K_{0}^{\prime}$ tel que $|\theta| \geq 1$, on ait $\left|\partial_{\xi_{j}} \dot{s}(x, \theta)\right| \leq(1 / 2 n M)$.

Quitte à restreindre à nouveau $K_{0}$, on peut supposer qu'il existe $c>0$ tel que, pour tout $\xi$ dans $K_{0} \cup-K_{0}$, et tout $\theta \in \mathbb{R}^{n}$ tel que $|\theta| \leq c|\xi|$, $\xi+\theta \in K_{0}^{\prime} \cup-K_{0}^{\prime}$. Une ultime restriction de $K_{0}$ permet de supposer, d'après (3.2) que l'on a, pour tout $(\xi, \eta) \in \widetilde{K},|\xi+\eta| \leq c|\xi|$; d'où pour tout $(\xi, \eta) \in \widetilde{K}$ tel que $|(\xi, \eta)| \geq 1$ on a :

$$
|b(x, \xi, \eta)| \leq \frac{1}{2}
$$

Soit $\chi \in \mathcal{C}^{\infty}\left(\mathbb{R}^{n}\right)$, homogène de degré 0 valant 1 sur $\bar{K}^{\prime}$, pour $|\xi| \geq 1$, supportée dans $\bar{K} \cap\left\{|\xi| \geq \frac{1}{2}\right\}$, on a pour tout $a_{ \pm} \in \mathrm{Op} S^{0}\left(\Omega_{0}\right)$ tel que $S E_{\left(a_{ \pm}\right)} \subset \Omega_{0} \times \pm K_{0}$

$$
\chi(\xi+\eta)\left(\xi_{n}+\eta_{n}\right) a_{+}(x, \xi) a_{-}(x, \eta)=r(x, \xi, \eta)\left(q_{1}(x, \xi)+q_{1}(x, \eta)\right),
$$

avec $r(x, \xi, \eta)=\left(\chi(\xi+\eta) a_{+}(x, \xi) a_{-}(x, \eta)\right) /(1-b(x, \xi, \eta))$. 
La deuxième étape consiste à démontrer que $r \in \Sigma_{\rho}^{0,0}\left(\Omega_{0}\right)$. La fonction $\tilde{b}: \widetilde{K} \rightarrow H_{\text {loc }}^{\rho+n / 2}\left(\Omega_{0}\right)$, qui à $(\xi, \eta)$ associe $b(x, \xi, \eta)$, est une fonction $\mathcal{C}^{\infty}$ $\operatorname{sur}\left\{(\xi, \eta) \mid(\xi+\eta) \geq \frac{1}{2}\right\}$.

D'après la formule de Faa di Bruno, il suffit de démontrer que l'on a, pour tout $t \in[0,1]$,

$$
\left|\partial_{\xi, \eta}^{\alpha} \partial_{\xi_{j}} s(x,-\xi+t(\xi+\eta))\right|_{\rho+n / 2} \leq C\langle\xi+\eta\rangle^{-|\alpha|} .
$$

Or $\mid \partial_{\xi, \eta}^{\alpha} \partial_{\xi_{j}} s\left(x,-\xi+\left.t(\xi+\eta)\right|_{\rho+n / 2} \leq C\langle-\xi+t(\xi+\eta)\rangle^{-|\alpha|}\right.$. Or, si $(\xi, \eta) \in \widetilde{K},|-\xi+t(\xi+\eta)| \approx|\xi|$, d'où le résultat.

Corollaire 3.2. - Les $G_{\sigma}$ et $\widetilde{G}_{\sigma}$ de la DÉfinition 1.2 sont fermés.

On va maintenant donner une traduction de ce lemme en termes d'opérateurs bilinéaires.

Corollaire 3.3. - Soient $Q \in \mathrm{Op} \underline{\Sigma}_{\rho}^{1}(\Omega),\left(x_{0}, \xi_{0}\right) \in T^{*} \Omega, K^{\prime}$ et $K$ deux ouverts coniques tels que $\sigma_{1}(q),\left(x_{0}, \xi_{0}\right), K^{\prime}$ et $K$ vérifient les hypothèses du LEMME 3.3 ; il existe alors : un voisinage ouvert $\Omega_{0}$ de $x_{0}$, un voisinage conique ouvert $K_{0}$ de $\Omega_{0}$, un opérateur pseudodifférentiel $\Lambda$ d'ordre 1 , elliptique sur $\Omega_{0} \times \bar{K}^{\prime}$, supporté dans $\Omega_{0} \times \stackrel{\circ}{K}$, tels que, si $N=[\rho]+1$, pour tout $R \in \mathrm{Op} \Sigma_{\rho}^{\mu, \mu^{\prime}}\left(\Omega_{0}\right)$ vérifiant

$$
\left\{\begin{array}{l}
\text { il existe un symbole } r \text { de } R \text { tel que } \\
\operatorname{Supp} r \subset\left\{(\xi, \eta) \in K_{0} \times-K_{0} \mid \xi+\eta \in K\right\}
\end{array}\right.
$$

on ait la propirété $\mathrm{L}_{\mathrm{N}}$ suivante

$$
\left\{\begin{array}{l}
\text { il existe }\left(R_{i, i^{\prime}}\right)_{0<i+i^{\prime} \leq N} \text { avec } R_{i, i^{\prime}} \in \mathrm{Op} \Sigma_{\rho-N+i+i^{\prime}}^{\mu+N-i-i^{\prime}-N+i+i^{\prime}}\left(\Omega_{0}\right) \\
\text { vérifiant }(L . S .) \text { tels que } \Lambda^{N} R=\sum_{0<i+i^{\prime} \leq N} R_{i, i^{\prime}}\left(Q^{i} \otimes Q^{i^{\prime}}\right) .
\end{array} .\right.
$$

Démonstration. - On va procéder par récurrence. La propriété $\left(\mathrm{L}_{1}\right)$ est vérifiée; c'est la traduction du lemme de l'hyperplan tangent en termes d'opérateurs. Si l'on suppose $\left(\mathrm{L}_{\mathrm{j}-1}\right)$ pour $j \leq N$, on a :

$$
\Lambda^{j} R=\sum_{0<i+i^{\prime} \leq j-1} \Lambda R_{i, i^{\prime}}\left(Q^{i} \otimes Q^{i^{\prime}}\right)
$$

l'application de $\left(\mathrm{L}_{1}\right)$ aux opérateurs $R_{i, i^{\prime}}$ assure alors $\left(\mathrm{L}_{\mathrm{j}}\right)$ et donc le résultat.

On est maintenant en mesure de démontrer la proposition clé qui donne les propriétés microlocales du reste.

$$
\text { TOME } 116-1988-\mathrm{N}^{\mathrm{O}} 3
$$


Proposition 3.2. - Soit u solution réelle $H_{\mathrm{loc}}^{s}(\Omega)$ de $(\mathrm{E})$ avec $s>\frac{1}{2} n+m+1-d$, alors pour tout $R \in \mathrm{Op} \Sigma_{s-n / 2-m-1+d}^{\mu, 0}(\Omega)$, avec $\mu \leq 2 s$, et tout $\sigma \leq 3 s-n-m-1+d, W F_{\sigma}(R(u, u)) \subset G_{\sigma+\mu}$.

Corollaire 3.4. - On obtient le ThÉORÈme 1.1.

Corollaire 3.5. - Soit $u$ solution réelle $H_{\mathrm{loc}}^{s}(\Omega)$ de $(\mathrm{E}), s>\frac{1}{2} n+$ $m+1-d, \sigma \leq 3 s-2 m-n+2 d-2$, on a alors :

$$
W F_{\sigma-m+1}\left(\mathrm{OP} P_{T}(p) \cdot u\right) \subset G_{\sigma+m+1-d} .
$$

Démonstration de la Proposition 3.2. - On va procéder par partition de l'unité microlocale de la manière suivante : soit $\left(x_{0}, \xi_{0}\right) \notin G_{\sigma+\mu}$ et soit $\left(\xi_{1}, \xi_{2}\right)$ dans $\left(\left.\operatorname{Car} p_{m}\right|_{x_{0}} \cap S^{n-1}\right)^{2}$.

Premier cas : $\xi_{1} \neq-\xi_{2}$ et $\xi_{0} \in \mathbb{R}_{+} \xi_{1}+\mathbb{R}_{+} \xi_{2}$.

Soit $\left(\sigma_{1}, \sigma_{2}\right)$ tel que $\sigma_{1}+\sigma_{2}=\sigma+\frac{1}{2} n+\mu$, d'après la Définition 1.2, pour $i$ valant 1 ou $2,\left.\xi_{i} \notin F_{\sigma_{i}}\right|_{x}$; donc il existe un $\Omega$ voisinage de $x_{0}$, des voisinages coniques $K_{i}$ de $\xi_{i}$ tels que $u \in H^{\sigma_{i}}\left(\Omega \times K_{i}\right)$.

Deuxième cas : $\xi_{1} \neq-\xi_{2}$ et $\xi_{0} \notin \mathbb{R}_{+} \xi_{1}+\mathbb{R}_{+} \xi_{2}$.

Il existe des voisinages coniques $K_{i}$ de $\xi_{i}$, un voisinage conique $K$ de $\xi_{0}$ tels que $\overline{K_{1}+K_{2}} \cap K=\emptyset$.

Troisième cas: $\xi_{1}=-\xi_{2}$ et $\xi_{1} \notin F_{\sigma_{2}}$.

Il existe un voisinage conique $K_{1}$ de $\xi_{1}$, voisinage $\Omega$ de $x_{0}$ tels que $u \in H^{1 / 2(\sigma+\mu+n / 2)}$ microlocalement $\operatorname{sur} \Omega \times K_{1} \cup-K_{1}$.

Quatrième cas : $\xi_{1} \in F_{\sigma_{2}}$.

On applique le Corollaire $3.3 \mathrm{du}$ lemme de l'hyperplan tangent. On a aussi construit une famille d'ouverts coniques $\left(K_{\xi, \eta}^{(1)} \times K_{\xi, \eta}^{(2)}\right)$ formant un recouvrement ouvert du compact $\left(\left.\operatorname{Car} p_{m}\right|_{x_{0}} \cap S^{n-1}\right)^{2}$. On en extrait un sous recouvrement fini; soit $\widetilde{\Omega}$ l'intersection de tous les voisinages de $x_{0}$ apparaissant alors, et soit $K_{0}$ l'intersection de tous les voisinages coniques de $\xi_{0}$ apparaissant.

En utilisant une partition de l'unité microlocale basée sur ce recouvrement de $\left(\operatorname{Car} p_{m} \cap S^{n-1}\right)^{2}$, les résultats d'ellipticité microlocale et les propriétés opératoires des opérateurs bilinéaires, on obtient le résultat.

Les deux corollaires résultent clairement de la proposition; signalons cependant pour le Corollaire 3.4 que, lorsque $\sigma \leq 3 s-2 m-n+2 d-2$, si $\sigma_{1}+\sigma_{2}=\sigma+\frac{1}{2} n+m+1-d$, vu que $u \in H_{\text {loc }}^{s}(\Omega)$ avec $s>\frac{1}{2} n+m+1-d$, on a $\sigma_{i} \leq 2 s-\frac{1}{2} n-m-1+d$, ce qui est exactement la zone couverte par le théorème de propagation de Bony (Voir [Bo.1]).

On peut maintenant démontrer le THÉORÈme 1.2. 
Soit $\sigma \in\left[2 s-\frac{1}{2} n-m-1+d, 3 s-n-2 m-2+2 d\right]$ et soit $\left(x_{0}, \xi_{0}\right)$ n'appartenant pas à $\operatorname{Car} p_{m} \cup G_{\sigma+m+1-d}$ (resp. $C$ une partie continue quelconque d'une bicaractéristique $\Gamma$ telle que $F_{\sigma} \cup H_{\sigma+m+1-d}=\emptyset$ ); il résulte du théorème d'ellipticité microlocale de Bony (resp. du théorème de propagation de Bony) qu'il existe un réel $\delta>0$, et deux opérateurs pseudodifférentiels d'ordre $0, A$ et $A^{\prime}$ tels que : $A$ soit elliptique près de $\left(x_{0}, \xi_{0}\right)$ (resp. de $\left.C\right), A^{\prime} u \in H^{2 s-(n / 2)-m+d}$ (resp. $H^{2 s-(n / 2)-m-1+d}$ ), pour tout $(x, \xi) \in S E(A), d\left(\xi /|\xi|,\left.S E\left(I-A^{\prime}\right)\right|_{x} \cap S^{n-1}\right) \geq 2 \delta$.

Soit $T$ un paraproduit tel que le (ii) de la Proposition 2.2 s'applique avec $\epsilon=\delta$; quitte à restreindre $S E(A)$, on peut, d'après le Corollaire 3.4 supposer que $\operatorname{AOPP}_{T}(p) \cdot u \in H^{\sigma-m+1}$, et donc, d'après la Proposition 2.2 (ii), $A O P P_{T}(p) \cdot A^{\prime} u \in H^{\sigma-m+1}$; le théorème d'ellipticité microlocale de Bony (resp. le théorème de propagation de Bony) assure alors que $u$ est $H^{\sigma+1}$ microlocalement près de $\left(x_{0}, \xi_{0}\right)$ (resp. $H^{\sigma}$ microlocalement près de $C$ ), d'où le théorème.

\section{4. Étude d'un exemple d'équation des ondes semi-linéaire à deux vitesses}

Le but est maintenant de démontrer, au travers de l'étude d'un exemple, que le ThÉorÈme 1.2 ainsi que les résultats qui en découlent sont essentiellement optimaux, c'est-à-dire que les phénomènes d'interaction que l'on prétend limiter se produisent effectivement, avec une intensité proche de la limite qui leur est fixée par le théorème.

On va considérer, pour $i$ valant 1 ou 2, les opérateurs suivants :

$$
\square_{i}=\frac{\partial^{2}}{\partial t^{2}}-c_{i}^{2}\left(\frac{\partial^{2}}{\partial x_{1}^{2}}+\frac{\partial^{2}}{\partial x_{2}^{2}}\right) \quad \text { avec } c_{1}>c_{2}
$$

On appellera rapide tout ce qui sera relatif à $\square_{1}$ et lent tout ce qui sera relatif à $\square_{2}$. On va démontrer le théorème suivant :

ThÉORÈme 4.1. - Soient $s$ et $\epsilon$ tels que $0<\epsilon<\frac{1}{2}\left(s-\frac{5}{2}\right)$; il existe $\beta \in \mathcal{C}_{0}^{\infty}\left(\mathbb{R}_{+}\right)$et $u \in H_{\mathrm{loc}}^{s}\left(\mathbb{R}^{3}\right)$ solution de $\square_{1} \square_{2} u=\beta u^{2}$ telles que :

(i) $W F(u) \cap(t<0)=N\left(C_{1}\right) \cap(t<0)$,

(ii) Supp $\operatorname{Sing}_{2 s+7 / 2+\epsilon}(u) \cap(t>0)=\left[C_{1}, C_{2}\right]$,

(iii) $u \in H^{3 s+1-\epsilon}\left(\stackrel{\circ}{C}_{2}\right)$.

Ce théorème est illustré géométriquement dans la Figure 4.1.

Remarquons que ce théorème légitime l'introduction de la notion d'onde lente et montre que lorsque l'hypersurface caractéristique dans le conormal de laquelle est localisé le front d'onde de la solution n'est pas lente, il apparaît des singularités du type $H^{2 s+s_{1}}$.

TOME $116-1988-\mathrm{N}^{\circ} 3$ 


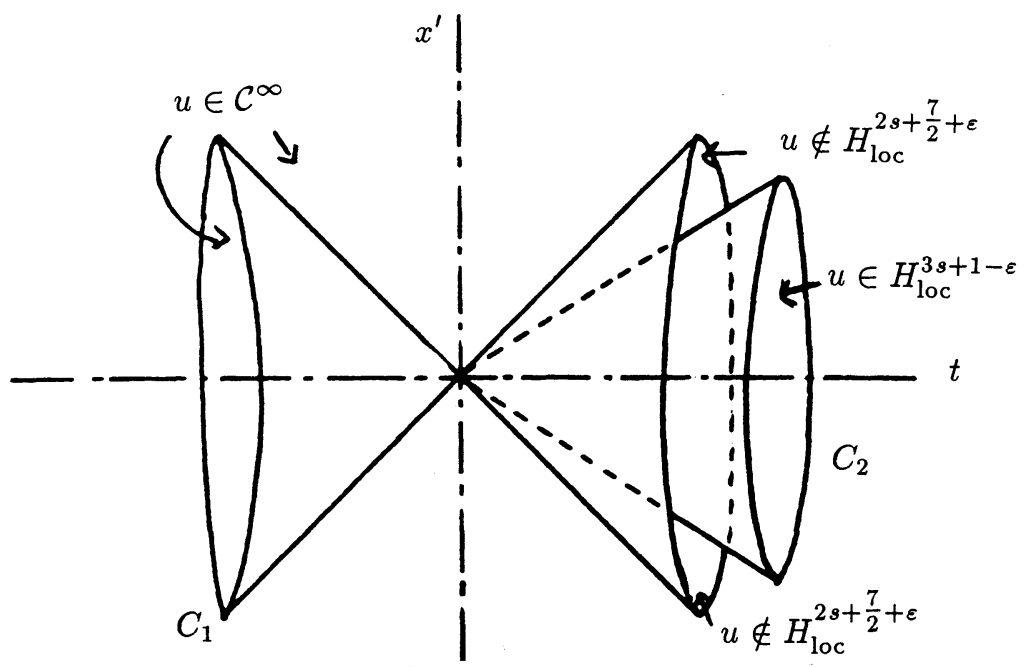

Fig. 4.1

On va maintenant mettre en place les outils nécessaires à la démonstration de ce théorème; ils sont construits à partir de ceux utilisés par M. Beals dans [4].

Définition 4.1. - Soient $s$ et $r$ deux réels tels que $s \leq r$ :

(i) $H^{s, r}\left(\square_{i}\right)=\left\{u \in \mathcal{S}^{\prime}|\langle\tau, \xi)\rangle^{s}\left\langle|\tau|-c_{i}|\xi|\right\rangle^{r-s} \hat{u}(\tau, \xi) \in L^{2}\left(\mathbb{R}^{3}\right)\right\}$,

(ii) $H^{s, r}=H^{s, r}\left(\square_{1}\right)+H^{s, r}\left(\square_{2}\right)$,

(iii) $H_{\text {loc }}^{s, r}=\left\{u \in \mathcal{D}^{\prime} \mid \forall \varphi \in \mathcal{C}_{0}^{\infty}, \varphi u \in H^{s, r}\right\}$.

Ces espaces sont des analogues des espaces $H^{s}(P)$ introduits précédemment. Mais, travaillant ici à coefficients constants, on peut, grâce à l'analyse de Fourier, définir et étudier des espaces plus précis. Cette précision supplémentaire est nécessaire à la démonstration du théorème.

Il convient maintenant d'introduire les nécessaires ajouts microlocaux.

Définition 4.2. - Soient $s, \bar{r}, r, g$ quatre réels tels que $s \leq \bar{r} \leq r \leq g$; soit $C_{1}$ le cône de lumière rapide issu de 0 ; on définit de $C_{1} \backslash\{0\}$ dans $S^{1}$ l'application $\omega$ qui, à $p=(t, x) \in C_{1} \backslash\{0\}$ associe $\omega(p)=-x / c_{1} t$. Posons $\Omega(p)=\mathbb{R}^{*}\left(c_{1}, \omega(p)\right)$ pour $p \in C_{1} \backslash\{0\}, \Omega(0)=\mathbb{R}^{3}$ et $\Omega(p)=\emptyset$ si $p \notin C_{1}$; $H_{\mathrm{loc}}^{s, r, \bar{r}, g}$ désigne l'ensemble des distributions $v$ de $H_{\text {loc }}^{s, r}$ telles que

(i) $v$ soit microlocalement $H^{g}$ aux points $(p ; \tau, \xi)$ tels que : ou bien $(\tau, \xi) \in \operatorname{Car} \square_{1}$ et $(\tau, \xi) \notin \Omega_{(p)}$, ou bien $(\tau, \xi) \in \operatorname{Car} \square_{2}$ et si $\Gamma_{2}$ désigne la bicaractéristique lente issue de $(p ; \tau, \xi)$, ou bien $\Gamma_{2} \cap C_{1}=\emptyset$, ou bien $\left\{p^{\prime}\right\}=\Gamma_{2} \cap C_{1}$ et alors $(\tau, \xi)$ n'appartient au plan tangent en $\eta$ à $\operatorname{Car} \square_{1}$ pour aucun $(\sigma, \eta) \in \Omega_{\left(p^{\prime}\right)}$.

(ii) $v$ est microlocalement $H^{\bar{r}}$ aux autres points caractéristiques lents. 
Cette définition rappelle, dans un cas très particulier, les Définitions 1.2 et 1.3 .

Définition 4.3. - Soit $\beta \in \mathcal{C}_{0}^{\infty}(\mathbb{R})$; soit $v \in \mathcal{S}^{\prime}$; on définit $E \beta$ par :

$$
\begin{aligned}
\mathcal{F}_{x}(E \beta v)(t, \xi) & =\frac{1}{c_{1}\left(c_{1}^{2}-c_{2}^{2}\right)|\xi|^{3}} \int_{-\infty}^{t} \sin \left(c_{1}(t-s)|\xi|\right) \beta(s) \mathcal{F}_{x}(w)(s, \xi) d s \\
& -\frac{1}{c_{2}\left(c_{1}^{2}-c_{2}^{2}\right)|\xi|^{3}} \int_{-\infty}^{t} \sin \left(c_{2}(t-s)|\xi|\right) \beta(s) \mathcal{F}_{x}(w)(s, \xi) d s
\end{aligned}
$$

Le théorème suivant, permet l'utilisation de ces outils.

THÉORÈME 4.2 .

(a) $E \beta$ envoie $H_{\mathrm{loc}}^{s, r, \bar{r}, g}$ dans $H_{\mathrm{loc}}^{s+3, r+4, \bar{r}+3, g+3}$.

(b) $S i \inf \left(s, s^{\prime}\right)>\frac{3}{2}$ et si $\operatorname{Inf}\left(r-s, r^{\prime}-s^{\prime}\right)>\frac{1}{2}$, alors

$$
H_{\mathrm{loc}}^{s, r, \bar{r}, g} \cdot H_{\mathrm{loc}}^{s^{\prime}, r^{\prime}, \bar{r}^{\prime}, g^{\prime}} \subset H_{\mathrm{loc}}^{s^{\prime \prime}, r^{\prime \prime}, \bar{r}^{\prime \prime}, g^{\prime \prime}},
$$

avec $s^{\prime \prime}=\operatorname{Inf}\left(s, s^{\prime}\right), \quad r^{\prime \prime}=\operatorname{Inf}\left(r, r^{\prime}, s+s^{\prime}-1-\epsilon\right)$,

$$
\begin{aligned}
& \bar{r}^{\prime \prime}=\operatorname{Inf}\left(\bar{r}, \bar{r}^{\prime}, s+s^{\prime}-1-\epsilon\right), \\
& g^{\prime \prime}=\operatorname{Inf}\left(g, g^{\prime}, \bar{r}^{\prime}+s-1, \bar{r}+s^{\prime}-1, \bar{r}+s-1, r+\bar{s}-1\right) .
\end{aligned}
$$

Démonstration. - La facile vérification de (a) est laissée au lecteur. Pour démontrer (b), on utilise une caractérisation de $H^{s, r}\left(\square_{i}\right)$ à l'aide de la théorie de Littlewood-Paley.

Soit $\varphi$ une fonction $\mathcal{C}^{\infty}$ supportée dans une couronne et réalisant une partition de l'unité dyadique; on a :

$$
u \in H^{s, r}\left(\square_{i}\right) \Longleftrightarrow\left|\left\langle|\tau|-c_{i}|\xi|\right\rangle^{r-s} \hat{u}_{q}\right|_{0} \leq c_{q} 2^{-q s} \quad \text { avec } \quad \sum c_{q}^{2} \leq C .
$$

On voit facilement que, pour obtenir $H^{s, r} \cdot H^{s^{\prime}, r^{\prime}} \subset H^{s^{\prime \prime}, r^{\prime \prime}}$, il suffit de démontrer que si $s_{1}>1, r_{1}-s_{1}>\frac{1}{2}$ et $r_{2}<s_{1}+s_{2}-1$, alors, tout paraproduit $T$ sur $\mathbb{R}^{3}$ envoie $H^{s_{1}, r_{1}}\left(\square_{j}\right) \times H^{s_{2}, r_{2}}\left(\square_{i}\right)$ dans $H^{s_{2}, r_{2}}\left(\square_{i}\right)$; en effet, posons $f_{q}=S_{q-N_{0}}(u) v_{q}$ : vu que $\left\langle|\tau|-c_{i}|\xi|\right\rangle \leq$ $\left\langle|\tau-\sigma|-c_{i}|\xi-\eta|\right\rangle+\langle\sigma, \eta\rangle$, on a :

Or

$$
\left|\left\langle|\tau|-c_{i}|\xi|\right\rangle \hat{f}_{q}\right|_{0} \leq \sum_{p \leq q-N_{0}}\left\|\langle\sigma, \eta\rangle^{r_{2}-s_{2}} \hat{u}_{p}\right\|_{L^{1}}\left|\left\langle|\tau|-c_{i}|\xi|\right\rangle^{r_{2}-s_{2}} \hat{v}_{q}\right|_{0} .
$$

$$
\left\|\langle\sigma, \eta\rangle^{r_{2}-s_{2}} \hat{u}_{p}\right\|_{L^{1}} \leq 2^{p\left(r_{2}-s_{2}\right)} \int \frac{\left\langle|\sigma|-c_{j}|\eta|\right\rangle^{r_{1}-s_{1}} \hat{u}_{p}(\sigma, \eta) d \sigma d \eta}{\left\langle|\sigma|-c_{j}|\eta|\right\rangle^{r_{1}-s_{1}}} .
$$

TOME $116-1988-\mathrm{N}^{\circ} 3$ 
D'après une inégalité de Schwarz et vu que $r_{1}-s_{1}>\frac{1}{2}$

$$
\left\|\langle\sigma, \eta\rangle^{r_{2}-s_{2}} \hat{u}_{p}\right\|_{L^{1}} \leq 2^{p\left(r_{2}-s_{2}-s_{1}-1\right)} .
$$

Par une localisation et par une partition de l'unité microlocale analogue à celle faite pour démontrer la Proposition 3.2 , on est ramené à démontrer le lemme suivant.

Lemme 4.1. - Soit $\left(\tau_{0}, \xi_{0}\right) \notin T_{\left(c_{1}, \omega\right)} \operatorname{Car} \square_{1}$; il existe un voisinage conique $K$ de $\left(c_{1}, \omega\right)$ tel que si $(u, v) \in H^{s, r}\left(\square_{1}\right) \times H^{s^{\prime}, r^{\prime}}\left(\square_{1}\right)$ et si $\operatorname{Sp}(u) \subset K$, et $\operatorname{Sp}(v) \subset-K$ alors uv est $H^{\gamma}$ microlocalement en $\left(\tau_{0}, \xi_{0}\right)$, avec $\gamma=\operatorname{Inf}\left(r^{\prime}+s-1, r+s^{\prime}-1\right)$.

Démonstration. - Nous renvoyons à [4] pour la démonstration de l'inégalité de convexité suivante : il existe un voisinage conique $K_{0}$ (resp. $K)$ de $\left(\tau_{0}, \xi_{0}\right)$ (resp. de $\left(c_{1}, \omega\right)$ ), et une constante $C>0$ tels que, pour tout $((\tau, \xi) ;(\sigma, \eta)) \in(-K) \times K$ tel que $(\tau+\sigma, \xi+\eta) \in K_{0}$ on ait :

$$
|\tau+\sigma, \xi+\eta| \leq C\left(\tau+c_{1}|\xi|+\left(\sigma-c_{1}|\eta|\right)\right)
$$

Remarquons que c'est cette inégalité qui est à l'origine du lemme de l'hyperplan tangent 3.3 .

La théorie de Littlewood-Paley donne alors trivialement le lemme et ainsi le théorème.

Le résultat clé pour la démonstration du THÉoRÈme 4.1 est la proposition suivante.

Proposition 4.1. - Soient $t_{0}, \epsilon$ et $s$ trois réels tels que $t_{0}<0$ et $0<\epsilon<\frac{1}{2}\left(s-\frac{5}{2}\right)$; il existe alors une solution $H^{s}$ de $\square_{1} v=0$ et une fonction $\beta \in \mathcal{C}_{0}^{\infty}(] t_{0},\left|t_{0}\right|[)$ telles que:

(i) $W F(v)=\left\{\left(t,-c_{1} t \omega ; r c_{1}, r \omega\right) \mid t \in \mathbb{R} ; r \in \mathbb{R}^{*}\right\}$

(ii) Il existe $\Gamma_{2}$ et $\Gamma_{2}^{\prime}$ deux bicaractéristiques lentes issues de points de type $\left(\tau,-c_{1} t \omega\right)$ avec $t$ dans $] t_{0},\left|\tau_{0}\right|\left[\right.$ et telles que $E \beta v^{2}$ ne soit pas $H^{2 s+(7 / 2)+\epsilon}$ microlocalement le long de $\Gamma_{2} \cup \Gamma_{2}^{\prime}$ pour $t \geq\left|t_{0}\right|$.

La figure 4.2 fournit une illustration géométrique de cette proposition.

Remarque. - La raison du décalage en $t_{0}<0$ est technique. On veut en effet rendre compte de ce qui se passe au voisinage d'un point du cône d'avenir avec des fonctions de localisation à transformée de Fourier positive pour pouvoir minorer commodément; l'origine est bien sûr le seul point justiciable d'un tel traitement.

Démonstration. - Elle est assez longue. Elle utilise la même fonction de base que celle de [4]. 


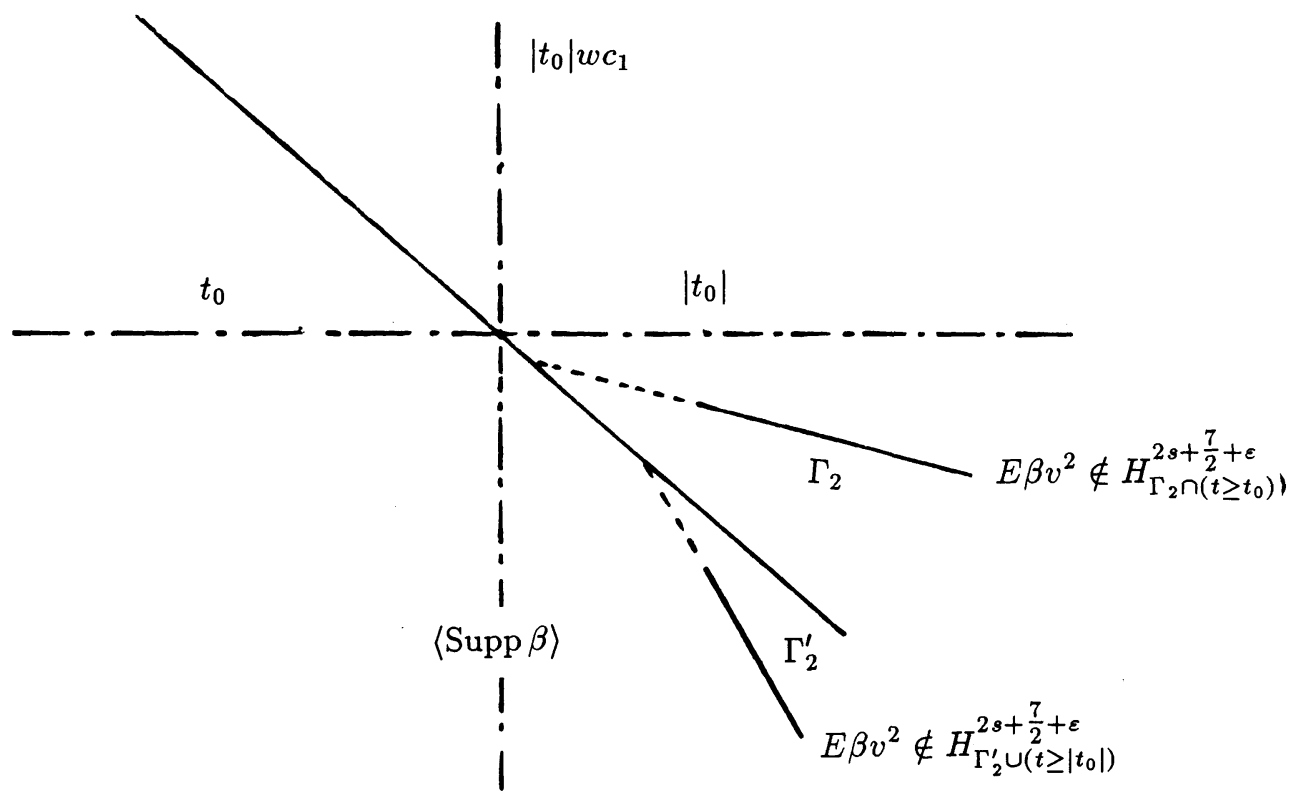

Fig. 4.2

Soit $\rho>1$, on pose $\sigma(\rho)=s+(s-1) \rho+2(\rho-1)$. Remarquons que $\sigma(\rho)>1$. Soient $\Phi \in \mathcal{C}_{0}^{\infty}\left(\mathbb{R}^{2}\right)$ telle que $\operatorname{Supp} \Phi$ soit un voisinage de 0 , $\Phi \geq 0, \hat{\Phi} \geq 0$ et $\hat{\Phi}(0)>0$, et $\omega \in S^{1}$ : posons

$$
g_{\rho}(x)=\sum_{k=1}^{+\infty} \frac{1}{k^{\sigma(\rho)}} \Phi(k x) e^{i\left(k^{\rho} x \mid \omega\right)}
$$

La singularité de $g_{\rho}$ en 0 est décrite par le lemme suivant.

LEMME 4.2.

(i) $W F(g)=\left\{(0, r \omega) \mid r \in \mathbb{R}_{+}^{*}\right\}$,

(ii) $0 \leq \hat{g}_{\rho}(\xi) \leq(C /\langle\xi\rangle)^{s+1+a(\rho)}$ avec $a(\rho)>0$ et $a(\rho)=O(\rho-1)$,

(iii) $s i|\xi /| \xi|-\omega| \leq C(\rho-1) /(\langle\xi\rangle \rho)$ alors $\hat{g}_{\rho}(\xi) \geq(C /\langle\xi\rangle)^{s+1+O(\rho-1)}$.

Pour la démonstration, nous renvoyons à [4].

Soit $v$ définie par :

$$
\mathcal{F}_{x}(v)(t, \xi)=e^{i t c_{1}|\xi|} \hat{g}_{\rho}(\xi)+e^{-i t c_{1}|\xi|} \hat{g}_{\rho}(-\xi) ;
$$

on a donc $\square_{1} v=0$ et donc $v \in H_{\text {loc }}^{s, \infty}\left(\mathbb{R}^{3}\right)$. Le lemme suivant va mettre en évidence les singularités de $v^{2}$ sur le plan tangent à $\operatorname{Car} \square_{1}$ en $\left(c_{1}, \omega\right)$.

TOME $116-1988-\mathrm{N}^{\circ} 3$ 
LEMME 4.3. - Soient $A_{0}, B_{0}, A, B$ quatre réels tels que :

$$
A_{0}<B_{0}, \quad A<B \quad \text { et } \quad 16 \leq \frac{A_{0}}{B}<\frac{B_{0}}{A}
$$

soit $\beta \in \mathcal{C}_{0}^{\infty}(] t_{0},\left|t_{0}\right|[)$ telle que $\hat{\beta} \geq 0$ et $\hat{\beta}(0)>0$; il existe une constante $C>0$ telle que si $(\tau, \xi)$ vérifie

$$
c_{1}^{2}|\xi|^{2}-\tau^{2} \in\left[A_{0}|\xi|^{2}, B_{0}|\xi|^{2}\right] \quad \text { et } \quad \tau-c_{1}(\xi \mid \omega) \in\left[\frac{1}{2} A|\xi|^{2-\rho}, \frac{1}{2} B|\xi|^{2-\rho}\right]
$$

alors $\widehat{\beta v}^{2}(\tau, \xi) \geq(2 C s) /\langle\xi\rangle+2+O(\rho-1)$.

Démonstration. - Vu le Lemme 4.2 et les propriétés de $\beta$ on a :

$$
\widehat{\beta v}^{2}(\tau, \xi) \geq m\left(D_{\iota, \xi}\right)(C /\langle\xi\rangle)^{2 s+2+O(\rho-1)},
$$

$D_{\tau, \xi}$ désignant l'ensemble des $\eta$ de $\mathbb{R}^{3}$ tels que :

$$
\begin{aligned}
& \left|\tau-c_{1}\right| \xi-\eta\left|+c_{1}\right| \eta|| \leq C_{1} \\
& || \xi-\eta\left|-c_{1} \omega\right| \xi-\eta|| \leq|\xi-\eta|^{1 / \rho} \\
& \left|\eta+c_{1}\right| \eta|\omega| \leq|\eta|^{1 / \rho} \quad \text { et } \quad \frac{A_{0}}{B}|\xi|^{\rho} \leq|\eta| \leq \frac{B_{0}}{A}|\xi|^{\rho} .
\end{aligned}
$$

Il s'agit donc de minorer la mesure de $D_{\tau, \xi}$. Si $\bar{\theta}$ désigne la projection orthogonale de $\theta$ sur l'orthogonal de $\omega, D_{\tau, \xi}$ contient l'ensemble $\underline{D}_{\tau, \xi}$ des $\eta$ tels que :

$$
\begin{aligned}
& \left|\tau-c_{1}\right| \xi-\eta\left|+c_{1}\right| \eta|| \leq C_{1}, \quad|\overline{\xi-\eta}| \leq 2|\xi| \\
& |\bar{\eta}| \leq 2|\xi| \quad \text { et } \quad \frac{A_{0}}{B}|\xi|^{\rho} \leq|\eta| \leq \frac{B_{0}}{A}|\xi|^{\rho} .
\end{aligned}
$$

Soit $\eta(\tau, \xi)=-\lambda(\tau, \xi) \omega$, avec

$$
\lambda_{(\tau, \xi)}=\frac{c_{1}^{2}|\xi|^{2}-\tau^{2}}{2\left(\tau-c_{1}(\xi \mid \omega)\right)}
$$

On a $\tau-c_{1}|\xi-\eta(\tau, \xi)|+c_{1}|\eta(\tau, \xi)|=0$. D'après (4.1) et (4.2) on a $\left(A_{0} / B\right)|\xi|^{\rho} \leq \lambda(\tau, \xi) \leq\left(B_{0} / A\right)|\xi|^{\rho}$. Or $\bar{\eta}(\tau, \xi)=0$, donc $\eta(\tau, \xi) \in \underline{D}_{\tau, \xi}$. D'autre part, il est clair qu'il existe $\delta>0$ tel que, pour tout $(\tau, \xi)$ tel que $|\xi| \geq 1, \eta(\tau, \xi)+B(0, \delta) \subset \underline{D}_{(\tau, \xi)}$, d'où le lemme.

Revenons à la démonstration de la Proposition 4.1 en $E \beta v^{2}$ sur $\left[\left|t_{0}\right|,+\infty\left[\right.\right.$. Soit $\gamma \in \mathcal{C}_{0}^{\infty}\left(\left[\left|t_{0}\right|,+\infty[)\right.\right.$; un calcul très simple assure que l'on a

$$
\mathcal{F}\left(\gamma E \beta v^{2}\right)(\tau, \xi)=\frac{1}{2 i\left(c_{2}^{2}-c_{1}^{2}\right)|\xi|^{3}}\left(\frac{P}{c_{2}}-\frac{Q}{c_{1}}\right),
$$

BULLETIN DE LA SOCIÉTÉ MATHÉMATIQUE DE FRANCE 
où l'on a posé, pour simplifier,

$$
\begin{aligned}
& P=\hat{\gamma}\left(\tau-c_{2}|\xi|\right) \widehat{\beta v}^{2}\left(c_{2}|\xi|, \xi\right)-\hat{\gamma}\left(\tau+c_{2}|\xi|\right) \widehat{\beta v}^{2}\left(-c_{2}|\xi|, \xi\right) \\
& Q=\hat{\gamma}\left(\tau-c_{1}|\xi|\right) \widehat{\beta v}^{2}\left(c_{1}|\xi|, \xi\right)-\hat{\gamma}\left(\tau+c_{1}|\xi|\right) \widehat{\beta v}^{2}\left(-c_{1}|\xi|, \xi\right)
\end{aligned}
$$

Soit $\chi \in \mathcal{C}^{\infty}\left(\mathbb{R}^{n}\right)$, homogène de degré 0 pour $|\xi| \geq 1$, à support dans un voisinage conique de $\left(c_{2}, \omega^{\prime}\right)$, valant 1 au voisinage de $\left(c_{2}, \omega^{\prime}\right)$ avec $\left(c_{2}, \omega^{\prime}\right) \in \operatorname{Car} \square_{2} \cap T_{\left(c_{1}, \omega\right)} \operatorname{Car} \square_{1}$. Or, $\chi(\tau, \xi) \gamma\left(\tau+c_{2}|\xi|\right)$ et $\chi(\tau, \xi) \gamma(\tau \pm$ $\left.c_{1}|\xi|\right)$ sont à décroissance rapide donc si $\gamma \in \beta v^{2}$ est $H^{a}$ microlocalement en $\pm\left(c_{2}, \omega^{\prime}\right)$, alors, en posant

$$
\left.\Delta_{a}(\tau, \xi)=\langle\xi\rangle^{a-3}\left|\hat{\gamma}\left(\tau-c_{2}|\xi|\right)\right| \widehat{\beta v}^{2}\left(c_{2}|\xi|, \xi\right)\right),
$$

on a $\int_{K^{\prime}} \Delta_{a}(\tau, \xi)^{2} d \tau d \xi<+\infty$, si $K^{\prime}$ désigne l'ensemble des $(\tau, \xi)$ tels que

$$
\left|\tau-c_{2}\right| \xi|| \leq \alpha|\xi| \quad \text { et } \quad\left|c_{2}\right| \xi\left|-c_{1}(\xi \mid \omega)\right| \leq \alpha|\xi|
$$

Posons $\sigma=\tau-c_{2}|\xi|$; soit $K$ l'ensemble des $\xi$ tels que $\left|c_{2}\right| \xi\left|-c_{1}(\xi \mid \omega)\right| \leq$ $\alpha|\xi|$; il vient alors :

$$
\int_{K^{\prime}} \Delta_{a}(\tau, \xi)^{2} d \tau d \xi \geq \int_{K}\left[\int_{0}^{\alpha|\xi|}|\hat{\gamma}(\sigma)|^{2} d \sigma\right]\langle\xi\rangle^{a-3} \widehat{\beta v}^{2}\left(c_{2}|\xi|, \xi\right) d \xi
$$

Il existe $C$ et $R>0$ tels que, si $|\xi| \geq R$, alors

$$
\int_{0}^{\alpha|\xi|}|\hat{\gamma}(\sigma)|^{2} d \sigma \geq C>0
$$

Soient $A_{0}$ et $B_{0}$ tels que $0<A_{0}<c_{1}^{2}-c_{2}^{2}<B_{0}$ et $A$ et $B$ vérifiant (4.1); si $K(R)$ désigne l'ensemble des $|\xi|$ tels que

$$
|\xi| \geq R \quad: \quad\left|c_{2}\right| \xi\left|-c_{1}(\xi \mid \omega)\right| \in\left[\frac{1}{2} A|\xi|^{2-\rho}, \frac{1}{2} B|\xi|^{2-\rho}\right]
$$

d'après le Lemme 4.3, et (4.3), on a

$$
\int_{K^{\prime}} \Delta_{a}^{2}(\tau, \xi) d \tau d \xi \geq C \int_{K(R)} \frac{d \xi}{\langle\xi\rangle^{2(2 s+5-a)+O(\rho-1)}} .
$$

On fait alors le changement de variables :

$$
r=|\xi|, \quad \theta_{1}=c_{2}|\xi|-c_{1}(\xi \mid \omega) .
$$

TOME $116-1988-\mathrm{N}^{\mathrm{O}} 3$ 
Son jacobien est clairement contrôlé d'où :

$$
\int_{K^{\prime}} \Delta_{a}^{2}(\tau, \xi) d \tau d \xi \geq \int_{R}^{+\infty}\left[\int_{-c_{2} r-B r^{2-\rho}}^{-c_{2} r-A r^{2-\rho}} d \theta_{1}\right] \frac{d r}{\langle r\rangle^{2(2 s+5-a+O(\rho-1))}} .
$$

Donc, si $\rho$ est assez proche de 1 , la projection en $(\tau, \xi)$ du front d'onde $H^{2 s+(7 / 2)+\epsilon}$ de $E \beta v^{2}$ contient $T_{\left(c_{1}, \omega\right)} \operatorname{Car} \square_{1} \cap \operatorname{Car} \square_{2}$.

D'autre part, supposons que, pour toute bicaractéristique $\Gamma_{2}$ issue de $\operatorname{Supp} \beta \times \mathbb{R}^{2} \cap \operatorname{Supp} \operatorname{Sing} v, E \beta v^{2}$ soit $H^{2 s+(7 / 2)+\epsilon}$ le long de $\Gamma_{2}$. Si $\Gamma_{2}^{\prime}$ est une bicaractéristique lente telle que $\operatorname{Supp} \beta \times \mathbb{R}^{3} \cap \operatorname{Supp} \operatorname{Sing} v \cap \Gamma_{2}^{\prime}=\emptyset$, le théorème de propagation de Hörmander assure $E \beta v^{2} \in \mathcal{C}^{\infty}\left(\Gamma_{2}^{\prime}\right)$, donc $E \beta v^{2} \in H^{2 s+(7 / 2)+\epsilon}$ ce qui est absurde; d'où la proposition.

Cette localisation est importante pour démontrer la proposition suivante :

Proposition 4.2. - Soient $s$ et $\epsilon$ tels que $0<\epsilon<\frac{1}{2}\left(s-\frac{5}{2}\right)$. Il existe $\beta \in \mathcal{C}_{0}^{\infty}\left(\mathbb{R}_{+}\right)$et $v \in H_{\mathrm{loc}}^{s}$ solution de $\square_{1} v=0$, telles que : $W F(v)=$ $N^{*}\left(C_{1}\right)$, si $\left(t_{0}, x_{0}\right)$ est tel que $\left|x_{0}\right|^{2} \in\left[c_{2}^{2} t_{0}^{2}, c_{1}^{2} t_{0}^{2}\right]$, si $\left(\tau_{2}, \xi_{2}\right) \in \operatorname{Car} \square_{2}$ est tel que $\left(x_{0} \mid \xi_{2}\right)+t_{0} \tau_{2}=0$; soit $\Gamma_{2}$ la bicaractéristique issue de $\left(t_{0}, x_{0} ; \tau_{2}, \xi_{2}\right) ;$ on a alors

$$
E \beta v^{2} \notin H^{2 s+(7 / 2)+\epsilon}\left(\Gamma_{2}\right) .
$$

Démonstration. - On suit la même démarche que dans [4]. Il suffit de translater et de dilater la Proposition 4.1, puis de condenser le tout à l'aide du théorème de Baire. Le fait que l'on remplisse l'entre deux cônes de singularités et seulement l'entre deux cônes, résulte de la remarque suivante : soit $\left(t_{0}, x_{0} ; \tau_{2}, \xi_{2}\right)$ un point caractéristique lent et soit $\left(t_{1}, x_{1}\right)$ le point d'intersection, supposé distinct de l'origine, entre $C_{1}$ et la bicaractéristique $\Gamma_{2}$ issue de $\left(t_{0}, x_{0} ; \tau_{2}, \xi_{2}\right)$. Un calcul facile laissé au lecteur montre l'équivalence des deux énoncés

$$
\left\{\begin{array}{l}
\left(\tau_{2}, \xi_{2}\right) \in T_{\left(c_{1},-\frac{x_{1}}{c_{1} t_{1}}\right)} \operatorname{Car} \square_{1} \cap \operatorname{Car} \square_{2} \\
x_{0} \xi_{2}+t_{0} \tau_{2}=0
\end{array}\right.
$$

On peut maintenant démontrer le THÉORÈme 4.1.

Soit $\beta$ et $v$ comme à la Proposition 4.2. On résoud le problème de Cauchy suivant :

$$
\square_{1} \square_{2} u=\beta u^{2}, \quad \gamma_{i} u=\gamma_{i} v \quad \text { avec } \quad i \in\{0,1,2,3\} .
$$

D'après le théorème d'existence des solutions d'un tel problème démontré dans [15], quitte à multiplier $v$ par une petite constante, on peut 
supposer la solution $u$ définie sur $\operatorname{Supp} \beta \times \mathbb{R}^{2}$; vu qu'alors $u=v+E \beta u^{2}$, $u$ est définie sur $\mathbb{R}^{3}$. D'après le ThÉORÈme 4.2 , on obtient par un gagne petit standard :

$$
u \in H_{\mathrm{loc}}^{s, 2 s+3-\epsilon, 2 s+2-\epsilon, 3 s+1-\epsilon} \text { pour tout } \epsilon>0 .
$$

On procède maintenant de manière classique : $u^{2}=v^{2}+2 v E \beta u^{2}+$ $\left(E \beta u^{2}\right)^{2}$. D'après le ThÉORÈme 4.2 que l'on utilisera systématiquement ici, on a $E \beta u^{2} \in H_{\text {loc }}^{s+3,2 s+3-\epsilon, 2 s+2-\epsilon, 3 s+1-\epsilon}$ ainsi que $\left(E \beta u^{2}\right)^{2}$. Vu que $v \in H_{\mathrm{loc}}^{s, \infty, \infty, \infty}$ on a $u^{2}-v^{2} \in H_{\mathrm{loc}}^{s, 2 s+3-\epsilon, 2 s+2-\epsilon, 3 s+1-\epsilon} \operatorname{donc} E \beta\left(u^{2}-v^{2}\right) \epsilon$ $H_{\text {loc }}^{s+3,2 s+7-\epsilon, 2 s+5-\epsilon, 3 s+4-\epsilon}$. Pour toute bicaractéristique lente $\Gamma$, on a donc :

$$
E \beta\left(u^{2}-v^{2}\right) \in H^{2 s+5-\epsilon}(\Gamma) .
$$

Soit alors $\left(t_{0}, x_{0} ; \tau_{2}, \xi_{2}\right)$ un point caractéristique lent tel que $\left(x_{0} \mid \xi_{2}\right)$ $+t_{0} \tau_{2}=0$, et soit $\Gamma$ la bicaractéristique issue de ce point. D'après la Proposition 4.2, on sait que

$$
E \beta v^{2} \notin H^{2 s+(7 / 2)+\epsilon}(\Gamma) .
$$

Or $u=v+E \beta u^{2}=v+E \beta v^{2}+E \beta\left(u^{2}-v^{2}\right)$.

\section{Appendice}

On se propose ici de démontrer le ThÉORÈme 2.2 qui dit que si $a \in \Sigma_{\rho}^{m, m^{\prime}}, b \in \underline{\Sigma}_{\rho}^{m_{1}}, c \in \underline{\Sigma}_{\rho}^{m_{2}}$ on a :

(i) pour tout $(s, t)$ tel que $s+t>m+\frac{1}{2} n$ (resp. $\left.s+t \geq m\right)$

$$
T_{b} \circ T_{a}-T_{b \# a} H^{s} \times H^{t} \longrightarrow H^{\sigma} \quad\left(\text { resp. } H_{-}^{\sigma}\right)
$$

avec $\sigma=s+t-\frac{1}{2} n-m-m^{\prime}-m_{1}+\rho$.

(ii) pour tout $(s, t)$ tel que $s+t>m+m_{1}+m_{2}+\frac{1}{2} n$ (resp. $\left.s+t \geq m+m_{1}+m_{2}\right)$

$$
\left.T_{a}\left(T_{b} \otimes T_{c}\right)-T_{a \#(b, c)} H^{s} \times H^{t} \longrightarrow H^{\sigma} \quad \text { (resp. } H^{\sigma}\right)
$$

avec $\sigma=s+t-\frac{1}{2} n-m-m^{\prime}-m_{1}-m_{2}+\rho$.

Démonstration

i) Il suffit de démontrer le résultat pour $a$ et $b$ vérifiant

$$
\begin{aligned}
\left|\partial_{\xi}^{\alpha} b(\cdot, \xi)\right|_{\rho-i+n / 2} & \leq\langle\xi\rangle^{m-i-|\alpha|}, \\
\left|\partial_{\xi, \eta}^{\alpha} a(\cdot, \xi, \eta)\right|_{\rho-j+n / 2} & \leq\langle\eta\rangle^{m}\langle\xi+\eta\rangle^{m^{\prime}-j-|\alpha|},
\end{aligned}
$$

TOME $116-1988-\mathrm{N}^{\circ} 3$ 
avec $i+j \leq[\rho]$. Comme lors de la démonstration du THÉORÈmE 2.1, on se ramène à des séries rapidement convergentes de symboles réduits pour $a$ et $b$, i.e. $a_{q}(x, \eta) \varphi_{q}(\xi+\eta)$ avec $\operatorname{Supp} \varphi_{q} \subset C_{q}$,

$$
\begin{aligned}
\left\|\varphi_{q}^{(\alpha)}\right\|_{0} & \leq C 2^{+q\left(m^{\prime}-|\alpha|\right)}, \\
\left|D^{\alpha} a_{q}(\cdot, \eta)\right|_{\rho-j+(n / 2)} & \leq C\langle\eta\rangle^{m} 2^{-q|\alpha|},
\end{aligned}
$$

et $b_{q^{\prime}}(x) \varphi_{1, q^{\prime}}(\xi)$, avec $\left\|\varphi_{1, q}^{(\alpha)}\right\|_{0} \leq C 2^{q\left(m_{1}-|\alpha|\right)}$, Supp $\varphi_{1, q} \subset C_{q}$ et $\left|b_{q^{\prime}}\right|_{\rho-i} \leq$ $C$, d'où, en posant $T_{q, q^{\prime}}(u, v)=T_{b_{q}} \varphi_{q}\left(2^{-q} D\right)\left\{T_{a_{q^{\prime}} \varphi_{q^{\prime}}}(u, v)\right\}$

$$
\begin{array}{r}
\mathcal{F}\left(T_{q, q^{\prime}}(u, v)\right)(\zeta)=\frac{1}{(2 \pi)^{2 n}} \sum_{\substack{r \geq q+N \\
|\nu| \leq N}} \int\left\{T_{1}(\zeta-\theta, \theta) T_{2}(\theta-\xi-\eta, \xi+\eta)\right. \\
\left.\quad \times \hat{b}_{q}(\zeta-\theta) \varphi_{1, q}(\theta) \hat{a}_{q^{\prime}}(\theta-\xi-\eta, \eta) \varphi_{q^{\prime}}(\xi+\eta) \hat{u}_{r}(\xi) \hat{v}_{r-\nu}(\eta)\right\} d \xi d \eta
\end{array}
$$

$\operatorname{Supp} T_{i} \subset\left\{\left(\theta_{1}, \theta_{2}\right) ;\left|\theta_{1}\right| \leq \epsilon_{i}\left|\theta_{2}\right|\right\}$ avec $\epsilon_{i}<\frac{1}{3}$, ce qui peut être supposé vu le THÉORÈme 2.1. On a alors :

$$
\begin{aligned}
|\zeta-\xi-\eta| & \leq|\zeta-\theta|+|\theta-\xi-\eta| \\
& \leq\left(\epsilon_{1} \epsilon_{2}+\epsilon_{1}+\epsilon_{2}\right)|\xi+\eta| \leq \frac{7}{9}|\xi+\eta| .
\end{aligned}
$$

Soit $T$ un paraproduit sur $\mathbb{R}^{n}$ tel que $T\left(\theta_{1}, \theta_{2}\right)$ vaille 1 au voisinage de $\left\{\left(\theta_{1}, \theta_{2}\right) ;\left|\theta_{1}\right| \leq \frac{7}{9}\left|\theta_{2}\right|\right\}$ d'où en posant

$$
X(\zeta, \theta, \xi, \eta)=T_{1}(\zeta-\theta, \theta) T_{2}(\theta-\xi-\eta, \xi-\eta)
$$

on a $T(\zeta-\xi-\eta, \xi+\eta) X=X$; il vient alors

$$
\begin{aligned}
\mathcal{F}\left(T_{q, q^{\prime}}(u, v)\right)(\zeta)= & \frac{1}{(2 \pi)^{2 n}} \sum_{\substack{r \geq q+N \\
|\nu| \leq N}} \int T(\zeta-\xi-\eta, \xi+\eta) \\
& \times \sigma_{q, q^{\prime}}(\zeta, \xi, \eta) \varphi_{q^{\prime}}(\xi+\eta) \hat{u}_{r}(\xi) \hat{v}_{r-\nu}(\eta) d \xi d \eta
\end{aligned}
$$

avec

$$
\sigma_{q, q^{\prime}}(\zeta, \xi, \eta)=\int X(\zeta, \theta, \xi, \eta) \hat{a}_{q^{\prime}}(\zeta-\theta-\xi-\eta, \eta) \hat{b}_{q}(\zeta, \theta) \varphi_{1, q}(\theta) d \theta
$$

En posant $\theta^{\prime}=\theta-\xi-\eta$, et en écrivant une formule de Taylor à l'ordre $M=[\rho]-i-j+1$ pour $\varphi_{1, q}$ entre $\xi+\eta$ et $\theta^{\prime}+\xi+\eta$ il vient $\sigma_{q, q^{\prime}}(\zeta, \xi, \eta)$ 
$=\sum_{\gamma=0}^{2} \sigma_{q}^{(\gamma)}(\zeta, \xi, \eta)$ avec

$$
\begin{aligned}
& \sigma_{q, q^{\prime}}^{(0)}(\zeta, \xi, \eta)=\left.\sum_{|\alpha| \leq M-1} \mathcal{F}_{x}\left(b_{q}(x) a_{q^{\prime}}(x, \eta)\right)\right|_{(\zeta-\xi-\eta)} \frac{1}{\alpha !} \varphi_{1, q^{(\xi+\eta)}}^{(\alpha)} \\
& \sigma_{q, q^{\prime}}^{(1)}(\zeta, \xi, \eta)= \sum_{|\alpha| \leq M-1} \int(1-X) \hat{b}_{q}\left(\zeta-\theta^{\prime}-\xi-\eta\right) \\
& \times \varphi_{1, q}^{(\alpha)}(\xi+\eta) \frac{\theta^{\prime \alpha}}{\alpha !} \hat{a}_{q^{\prime}}\left(\theta^{\prime}, \eta\right) d \theta^{\prime} \\
& \sigma_{q, q^{\prime}}^{(2)}(\zeta, \xi, \eta)= \sum_{|\alpha|=M} \iint_{0}^{1} X \hat{b}_{q}\left(\zeta-\theta^{\prime}-\xi-\eta\right) \varphi_{1, q}^{(\alpha)}\left(\xi+\eta+t \theta^{\prime}\right) \\
& \times \theta^{\prime \alpha} \hat{a}_{q^{\prime}}\left(\theta^{\prime}, \eta\right) \frac{(1-t)^{|\alpha|}}{\alpha !} d \theta^{\prime} d t .
\end{aligned}
$$

Remarquons que $\sigma_{q, q^{\prime}}^{(0)}(\zeta, \xi, \eta)=\mathcal{F}_{x}\left(b_{q} \varphi_{1, q} \# a_{q} \varphi_{q}\right) \mid(\zeta-\xi-\eta, \xi, \eta)$, et que $\sigma_{q, q^{\prime}}^{(1)}$ correspond au terme $S_{q}(a) S_{q}(b)-S_{q}(a b)$ dans la construction du calcul paradifférentiel linéaire.

Reste à étudier $T_{q^{\prime}}^{(\gamma)}(u, v)$ avec $\gamma \in\{1,2\}$ et

$$
\begin{array}{r}
\left(T_{q^{\prime}}^{(\gamma)}(u, v)\right)(\zeta)=\sum_{\substack{r \geq q+N \\
|\nu| \leq N,\left|\nu^{\prime}\right| \leq N}} \int T(\zeta-\xi-\eta, \xi+\eta) \sigma_{q^{\prime}-\nu, q^{\prime}}^{(\gamma)}(\zeta, \xi, \eta) \\
\times \varphi_{q^{\prime}}(\xi+\eta) \hat{u}_{r}(\xi) \hat{v}_{r-\nu}(\eta) d \xi d \eta
\end{array}
$$

car il est clair que $\left|q-q^{\prime}\right| \leq N$ dans les définitions de $T_{q^{\prime}}$.

Il est clair que $\operatorname{Sp}\left(T_{q^{\prime}}^{(\gamma)}(u, v)\right) \subset C_{q}^{\prime}$. Reste à majorer leurs normes $L^{2}$ pour démontrer que l'on a

$$
\sum_{q, q^{\prime}} T_{q, q^{\prime}}(u, v)-T_{b \# a}(u, v) \in H^{s+t-(n / 2)-m-m_{1}-m^{\prime}+\rho} .
$$

Majorons $\left|T_{q^{\prime}}^{(1)}(u, v)\right|_{0}$. Sur Supp $(1-X)$, on a ou bien $\left|\zeta-\theta^{\prime}-\xi-\eta\right| \geq$ $\tilde{\epsilon}|\xi+\eta|$ ou bien $\left|\theta^{\prime}\right| \geq|\xi+\eta|$; d'où, en découpant spectralement $a_{q^{\prime}}(x, \eta)$ ou bien $b_{q}(x)$ on a :

$$
\sigma_{q, q^{\prime}}^{(1)}(\zeta, \xi, \eta) \leq h_{q, q^{\prime}}^{(1)}(\zeta-\xi-\eta, \eta)+h_{q, q^{\prime}}^{(2)}(\zeta-\xi-\eta, \eta)
$$

avec

$$
\begin{aligned}
& h_{q, q^{\prime}}^{(1)}(\sigma, \eta)=\sum_{\substack{|\alpha| \leq M-1 \\
|p-q| \leq N}} 2^{q^{\prime}\left(m_{1}-i-|\alpha|\right)} \times \int\left|\varphi\left(2^{-p}(\sigma-\theta)\right) b_{q}(\sigma-\theta) \theta^{\alpha} \hat{a}_{q^{\prime}}(\theta, \eta)\right| d \theta \\
& h_{q, q^{\prime}}^{(2)}(\sigma, \eta)=\sum_{\substack{|\alpha| \leq M-1 \\
\left|p-q^{\prime}\right| \leq N}} 2^{q^{\prime}\left(m_{1}-i-|\alpha|\right)} \int\left|b_{q}(\sigma-\theta) \varphi\left(2^{-p} \theta\right) \theta^{\alpha} \hat{a}_{q^{\prime}}(\theta, \eta)\right| d \theta . \\
& \text { TOME } 116-1988-\mathrm{N}^{\mathrm{o}} 3
\end{aligned}
$$


On a donc, vu les régularités respectives de $b_{q}$ et $a_{q^{\prime}}(\cdot, \eta)$ :

$\forall i \in\{1,2\}, \forall\left|\nu^{\prime}\right| \leq N \quad \operatorname{Sup}_{\eta \in C_{r}}\left\|h_{q^{\prime}-\nu, q^{\prime}}^{(i)}(\cdot, \eta)\right\|_{L^{1}} \leq C 2^{-q^{\prime}\left(\rho-m_{1}\right)} 2^{r m}$.

L'application du Lemme 2.1 avec

$$
h_{q^{\prime}}=\sum_{\left|\nu^{\prime}\right| \leq N} h_{q^{\prime}-\nu, q^{\prime}}^{(1)}+h_{q^{\prime}-\nu, q^{\prime}}^{(2)}
$$

$\Psi_{q^{\prime}}=\left|\varphi_{q^{\prime}}\right|, f_{r}=\left|\hat{u}_{r}\right|$ et $g_{r}=\sum_{|\nu| \leq N}\left|\hat{v}_{r}\right|$, assure :

$$
\left|R_{q^{\prime}}^{(1)}(u, v)\right|_{0} \leq c_{q^{\prime}} 2^{-q^{\prime}\left(s+t-(n / 2)-m-m^{\prime}-m_{1}+\rho\right)}
$$

$\forall(s, t)$ tel que l'on a $s+t \geq m$; et ce avec $\sum_{q} c_{q}^{2} \leq C$.

Majorons $\left|T_{q^{\prime}}^{(2)}(u, v)\right|_{0}$.

Si $1 \leq i, j+|\alpha| \leq[\rho]<\rho$, donc $\theta^{\alpha} \hat{a}(\theta, \eta) \in L^{1}(d \theta)$. De plus, on a :

$$
\left|\varphi_{1, q}^{(\alpha)}(t \theta+\xi+\eta)\right| \leq C 2^{q\left(m_{1}-|\alpha|-i\right)}
$$

d'où $\left|\sigma_{q, q^{\prime}}^{(2)}(\zeta, \xi, \eta)\right| \leq h_{q, q^{\prime}}(\zeta-\xi-\eta, \eta)$ avec

$$
\begin{gathered}
h_{q, q^{\prime}}(\sigma, \eta)=\sum_{|\alpha|=M} 2^{q\left(m_{1}-|\alpha|-i\right)} \int \sum_{\left|\nu^{\prime}\right| \leq N} \hat{b}_{q}(\sigma-\theta) \theta^{\alpha} \hat{a}_{q^{\prime}}(\theta, \eta) d \theta, \\
\operatorname{Sup}_{\eta \in K_{r}}\left\|h_{q^{\prime}-\nu, q^{\prime}}(\cdot, \eta)\right\|_{L^{1}} \leq C 2^{r m} 2^{-q^{\prime}\left(m_{1}-|\alpha|-i\right)} .
\end{gathered}
$$

On applique le Lemme 2.1 avec :

$$
\begin{aligned}
& h_{q^{\prime}}(\sigma, \eta)=\sum_{|\nu| \leq N} h_{q^{\prime}-\nu, q^{\prime}}(\sigma, \eta) ; \quad \Psi_{q^{\prime}}=\left|\varphi_{q^{\prime}}\right| ; \\
& f_{r}=\left|\hat{u}_{r}\right| ; \quad g_{r}=\sum_{|\nu| \leq N}\left|\hat{v}_{r-\nu}\right| ;
\end{aligned}
$$

d'où $\quad\left|R_{q^{\prime}}^{(2)}(u, v)\right|_{0} \leq c_{q^{\prime}} 2^{-q^{\prime}\left(s+t-(n / 2)-m-m_{1}-m^{\prime}+|\alpha|+i+j\right)}$

avec $\sum_{q} c_{q}^{2} \leq C|u|_{s}|v|_{t}$, or $|\alpha|+i+j=[\rho]+1>\rho$. 
Si $i=0, j+|\alpha|=[\rho]+1$ et alors $\theta^{\prime \alpha} \hat{a}_{q}\left(\theta^{\prime}, \eta\right) \notin L^{1}\left(d \theta^{\prime}\right)$. On découpe spectralement $a_{q}(\cdot, \eta)$ d'où $\left|\sigma_{q, q^{\prime}}^{(2)}(\zeta, \xi, \eta)\right| \leq h_{q, q^{\prime}}(\zeta-\xi-\eta, \eta)$ avec

$$
\begin{aligned}
& h_{q, q^{\prime}}(\sigma, \eta)=\sum_{\substack{|\nu| \leq N \\
p^{\prime} \leq q^{\prime}+N}} 2^{q^{\prime}\left(m_{1}-[\rho]+j-1\right)} \\
& \quad \times \int\left|\hat{b}_{q}(\sigma-\theta) \varphi\left(2^{-p} \theta\right)\right||\theta|^{[\rho]-j+1}\left|\hat{a}_{q}(\theta, \eta)\right| d \theta .
\end{aligned}
$$

Vu que $[\rho]-j-|\alpha|<0$

$$
\operatorname{Sup}_{\substack{\eta \in C_{r} \\|\nu| \leq N}}\left\|h_{q^{\prime}-\nu, q^{\prime}}(\cdot, \eta)\right\|_{L^{1}} \leq C 2^{r m} 2^{-q(\rho-j-|\alpha|)}
$$

d'où, comme précédemment, l'application du Lemme 2.1 assure

$$
\left|T_{q^{\prime}}^{(2)}(u, v)\right|_{0} \leq c_{q^{\prime}} 2^{-q^{\prime}\left(s+t-(n / 2)-m-m^{\prime}-m_{1}+\rho\right)}
$$

avec $\sum c_{q^{\prime}}^{2} \leq C|u|_{s}|v|_{t}$ d'où (i).

Démontrons maintenant (ii).

Comme précédemment, il suffit de démontrer le résultat pour $a, b, c$ vérifiant

$$
\begin{aligned}
& \left|\partial_{\xi, \eta}^{\alpha} a(\cdot, \xi, \eta)\right|_{\rho-i+(n / 2)} \leq C\langle\eta\rangle^{m}\langle\xi+\eta\rangle^{m^{\prime}-i-|\alpha|} \\
& \left|\partial_{\xi}^{\alpha} b(\cdot, \xi)\right|_{\rho-j+(n / 2)} \leq C\langle\xi\rangle^{m_{1}-j-|\alpha|} \\
& \left|\partial_{\xi}^{\alpha} c(\cdot, \xi)\right|_{\rho-\ell+(n / 2)} \leq C\langle\xi\rangle^{m_{2}-\ell-|\alpha|}
\end{aligned}
$$

Comme précédemment, il suffit de considérer les symboles réduits; on se ramène donc à considérer $a_{q}(x, \eta) \varphi_{q}(\xi+\eta)$ avec

$$
\begin{gathered}
\left\|\varphi_{q}^{(\alpha)}\right\|_{0} \leq C 2^{q(m-|\alpha|)}, \quad \operatorname{Supp} \varphi_{q} \subset C_{q}, \\
\left|D_{\eta}^{\alpha} a(\cdot, \eta)\right|_{\rho-i+(n / 2)} \leq C 2^{-q|\alpha|}\langle\eta\rangle^{m},
\end{gathered}
$$

$b_{q}(x) \varphi_{1, q}(\xi)$ avec $\left\|\varphi_{1, q}^{(\alpha)}\right\|_{0} \leq C 2^{q\left(m_{1}-|\alpha|\right)}$ et $\left|b_{q}\right|_{\rho-j+(n / 2)} \leq C$ et $c_{q}(x) \varphi_{2, q}(\xi)$ avec $\left\|\varphi_{2, q}^{(\alpha)}\right\|_{0} \leq C 2^{q\left(m_{2}-|\alpha|\right)}$ et $\left|c_{q}\right|_{\rho-\ell+(n / 2)} \leq C$

$$
\begin{aligned}
\mathcal{F}\left(T_{q}(u, v)\right)(\zeta)= & \sum_{\substack{r \geq q+N \\
|\nu| \leq N}} \int\left\{\widetilde{X} \hat{a}_{q}(\zeta-\xi-\eta, \eta) \varphi_{q}(\xi+\eta) \hat{b}_{r}(\xi-\theta)\right. \\
& \left.\times \hat{c}_{r-\nu}(\nu-\sigma) \varphi_{1, r}(\theta) \hat{u}(\theta) \varphi_{2, r-\nu}(\sigma) \hat{v}(\sigma)\right\} \frac{d \xi d \eta d \sigma d \theta}{(2 \pi)^{-4 n}}
\end{aligned}
$$

TOME $116-1988-\mathrm{N}^{\circ} 3$ 
avec $\tilde{X}(\zeta, \theta, \sigma, \xi, \eta)=T_{0}(\zeta-\xi-\eta, \xi+\eta) T_{1}(\xi-\theta, \theta) T_{2}(\eta-\sigma, \sigma)$

avec $\quad \operatorname{Supp} T_{i} \subset\left\{\left(\theta_{1}, \theta_{2}\right)|| \theta_{1}\left|\leq \epsilon_{i}\right| \theta_{2} \mid\right\}$

$$
\operatorname{Supp}\left(1-T_{i}\right) \subset\left\{\left(\theta_{1}, \theta_{2}\right)|| \theta_{1}\left|\geq \tilde{\epsilon}_{i}\right| \theta_{2} \mid\right\}
$$

avec $0<\tilde{\epsilon}_{i}<\epsilon_{i}<1$.

Une difficulté survient : sur Supp $\widetilde{X}$, on n'a pas, comme précédemment la bonne localisation spectrale, c'est-à-dire

$$
|\zeta-\theta-\sigma| \leq \epsilon|\theta+\sigma| \text { pour } \epsilon<1 \text {. }
$$

Pour obtenir cela, il faut procéder à une troncature dont on montrera bien sûr qu'elle est négligeable, c'est-à-dire $\rho$-régularisante.

Soit $1>\epsilon>0$ tel que $0<\left(\epsilon_{0}+2 \epsilon\right) /(1-2 \epsilon)<1$. Soit $\widetilde{T}$ un paraproduit tel que Supp $\widetilde{T} \subset\left\{\left(\theta_{1}, \theta_{2}\right) ;\left|\theta_{1}\right| \leq \epsilon\left|\theta_{2}\right|\right\}$. Posons $X(\zeta, \theta, \sigma, \xi, \eta)=$ $\widetilde{X}(\zeta, \theta, \sigma, \xi, \eta) \widetilde{T}(\xi-\theta, \xi+\eta) \widetilde{T}(\eta-\sigma, \xi+\eta)$. Sur Supp $X$ on a $|\theta+\sigma| \approx|\xi+\eta|$ et $|\zeta-\theta-\sigma| \leq\left(\epsilon_{0}+2 \epsilon\right) /(1-2 \epsilon)|\theta+\sigma|$. En effet on a $|\xi+\eta-\theta-\sigma| \leq 2 \epsilon|\xi+\eta|$ donc $|\theta+\sigma| \approx|\xi+\eta|$. De plus

$$
\begin{aligned}
|\xi-\theta-\sigma| & \leq|\zeta-\xi-\eta|+|\xi+\eta-\theta-\sigma| \\
& \leq\left(\epsilon_{0}+2 \epsilon\right)|\xi+\eta| \\
& \leq \frac{\epsilon_{0}+2 \epsilon}{1-2 \epsilon}|\theta+\sigma| .
\end{aligned}
$$

Soit $T$ un paraproduit sur $\mathbb{R}^{N}$ tel que $T$ vaille 1 sur $\left\{\left(\theta_{1}, \theta_{2}\right) ;\left|\theta_{1}\right| \leq\right.$ $\left.\left(\epsilon_{0}+2 \epsilon\right) /(1-2 \epsilon)\left|\theta_{2}\right|\right\}$. On a alors

$$
T(\zeta-\theta-\sigma, \theta+\sigma) X=X \text {. }
$$

De plus, on choisit $\epsilon$ assez petit de manière à ce qu'il existe $\lambda \in \mathcal{C}_{0}^{\infty}(K)$, $K$ couronne de $\mathbb{R}^{n}$ telle que

$$
\lambda\left(2^{-q}(\theta+\sigma)\right) X \varphi_{q}(\xi+\eta)=X \varphi_{q}(\xi+\eta) .
$$

En faisant apparaître $X$, puis $T$, puis en posant $\xi^{\prime}=\xi-\theta$ et $\eta^{\prime}=\eta-\sigma$, puis enfin en écrivant une formule de Taylor à l'ordre $M=[\rho]-i-j-\ell+1$ entre $(\theta, \sigma)$ et $(\xi+\theta, \eta+\sigma)$ on a

(i) $T_{q}(u, v)=\sum_{\gamma=0}^{3} T_{q}^{(\gamma)}(u, v)$ avec

$$
\begin{aligned}
\left(T_{q}^{(0)}(u, v)\right)(\zeta)=\sum_{\substack{r \geq q+N \\
|\nu| \leq N}} \int T(\zeta-\theta-\sigma, \theta+\sigma) \sigma_{q, r, \nu}^{(0)}(\zeta-\theta-\sigma, \theta, \sigma) \varphi_{1, r}(\theta) \\
\times \hat{u}(\theta) \varphi_{2, r^{\prime}-\nu} \hat{v}(\eta) \frac{d \xi d \eta}{(2 \pi)^{+2 n}} \\
\sigma_{q, r, \nu}^{(0)}(\tau, \theta, \sigma)=\mathcal{F}_{x}\left[\sum_{\left|\alpha_{1}+\alpha_{2}\right| \leq M-1} \partial_{\beta}^{\alpha_{1}} \partial_{\sigma}^{\alpha_{2}} a_{q}(x, \sigma) \varphi_{q}(\theta+\sigma)\right. \\
\left.\times D^{\alpha_{1}} b_{r}(x) D^{\alpha_{2}} c_{r-\nu}(x)\right](\tau) .
\end{aligned}
$$

BULLETIN DE LA SOCIÉTÉ MATHÉMATIQUE DE FRANCE 


$$
\begin{aligned}
& \text { (ii) }\left(T_{q}^{1}(u, v)\right)(\zeta)=\sum_{\substack{r \geq q+N \\
|\nu| \leq N}} \int(X-\tilde{X}) \hat{a}_{q}(\zeta-\xi-\eta, \eta) \varphi_{q}(\xi+\eta) \hat{b}_{r}(\xi-\theta) \\
& \times \hat{c}_{r-\nu}(\eta-\sigma) \varphi_{1, r}(\theta) \hat{u}(\theta) \varphi_{2, r-\nu} \hat{v}(\sigma) \frac{d \sigma d \theta d \xi d \eta}{(2 \pi)^{-4 n}} \\
& \begin{aligned}
\mathcal{F}\left(T_{q}^{(\gamma)}(u, v)\right)(\zeta)= & \sum_{\substack{r \geq q+N \\
|\nu| \leq N}} \int T(\zeta-\theta-\sigma, \theta+\sigma) \sigma_{q, r, \nu}^{(\gamma)}(\zeta-\theta-\sigma) \lambda_{q}(\theta+\sigma) \\
& \times \varphi_{1, r}(\theta) \hat{u}(\theta) \varphi_{2, r-\nu}(\sigma) \hat{v}(\sigma) \frac{d \theta d \sigma}{(2 \pi)^{+4 n}}
\end{aligned}
\end{aligned}
$$

où $\sigma_{q, r, \nu}^{(2)}(\zeta, \theta, \sigma)$ et $\sigma_{q, r, \nu}^{(3)}(\zeta, \theta, \sigma)$ sont donnés par les formules suivantes

$$
\begin{array}{r}
\sigma_{q, r, \nu}^{(2)}(\zeta, \theta, \sigma)=\sum_{\left|\alpha_{1}+\alpha_{2}\right| \leq M-1} \int d \xi^{\prime} d \eta^{\prime} \partial_{\lambda}^{\alpha_{1}} \partial_{\mu}^{\alpha_{2}}\left[\hat{a}_{q}\left(\zeta-\xi^{\prime}-\eta^{\prime}-\theta-\sigma, \mu\right)\right. \\
\left.\times \varphi_{q}(\lambda+\mu)\right]_{(\lambda, \mu)=(\theta, \sigma)} \xi_{1}^{\alpha_{1}} \hat{b}_{r}\left(\xi^{\prime}\right) \eta^{\prime \alpha_{2}} \hat{c}_{r-\nu}\left(\eta^{\prime}\right)(1-X) \\
\sigma_{q, r, \nu}^{(3)}(\zeta, \theta, \sigma)=\sum_{\left|\alpha_{1}+\alpha_{2}\right| \leq M} \iint_{0}^{1} d \xi^{\prime} d \eta^{\prime} d t\left\{\partial_{\lambda}^{\alpha_{1}} \partial_{\mu}^{\alpha_{2}} \hat{a}_{q}\left(\zeta-\xi^{\prime}-\eta^{\prime}-\theta-\sigma, \mu\right)\right. \\
\left.\left.\times \varphi_{q}(\lambda+\mu)\right)_{\theta+t \xi^{\prime}, \sigma+t \eta^{\prime}} \xi^{\prime \alpha_{1}} \hat{b}_{r}\left(\xi^{\prime}\right) \eta^{\prime \alpha_{2}} \hat{c}_{r-\nu}\left(\eta^{\prime}\right) X \frac{(1-t)^{M-1}}{(M-1) !}\right\}
\end{array}
$$

La sommation des termes du type $T_{q}^{0}(u, v)$ donne $T_{a \#(b, c)}(u, v)$. D'autre part, il est clair que $\operatorname{Sp}\left(T_{q}^{(\gamma)}(u, v)\right) \subset C_{q}^{\prime}$ pour tout $\gamma \in\{1,2,3\}$.

Reste donc à majorer $\left|T_{q}^{(\gamma)}(u, v)\right|_{0}$.

Premier cas : $\gamma=1$. Vu la définition de $X$ et de $\widetilde{X}$, en découpant spectralement $b_{r}$ et $c_{r-\nu}$ on a :

$$
\begin{aligned}
\left|T_{q}^{(1)}(u, v)(\zeta)\right| \leq \frac{1}{2^{q(\rho+i-\operatorname{Inf}(j, \ell))}} \sum_{\substack{r \geq q+N \\
\lambda+\mu=1}} \int \hat{a}_{q}(\zeta-\xi-\eta, \eta) \varphi_{q}(\xi+\eta) \\
\quad \times f_{r}^{(\lambda)}(\xi) g_{r}^{(\mu)}(\nu) d \xi d \eta
\end{aligned}
$$

les fonctions $f_{r}^{i}$ et $g_{r}^{i}$ étant données par les formules

$$
\begin{aligned}
& f_{r}^{(0)}=2^{r\left(m_{1}-j\right)}\left|\hat{u}_{r}\right|, \quad g_{r}^{(0)}=2^{r\left(m_{2}-\ell\right)} \sum_{|\nu| \leq N}\left|\hat{v}_{r-\nu}\right|, \\
& f_{r}^{(1)}=\operatorname{Sup}_{q \leq r-N} 2^{-q(\rho-j)}\left|\left(\Psi\left(2^{-r+N_{0}}\right)-\Psi\left(2^{-q+N_{0}}\right)\right) \hat{b}_{r}\right| 2^{r\left(m_{1}-j\right)}\left|\hat{u}_{r}\right|, \\
& g_{r}^{(1)}=\operatorname{Sup}_{q \leq r-N} 2^{-q(\rho-\ell)}\left|\Psi\left(2^{-r+N_{0}}\right)-\Psi\left(2^{-q+N_{0}}\right) \hat{c}_{r-\nu}\right| \\
& \times 2^{r\left(m_{1}-\ell\right)} \sum_{|\nu| \leq N}\left|\hat{v}_{r-\nu}\right| .
\end{aligned}
$$

TOME $116-1988-\mathrm{N}^{\circ} 3$ 
On applique le Lemme 2.1 d'où, vu que $i+j+\ell+\rho-\operatorname{Inf}(j, \ell) \geq \rho$, on a :

$$
\left|T_{q}^{(1)}(u, v)\right|_{0} \leq c_{q} 2^{-q\left(s+t-(n / 2)-m-m_{1}-m_{2}-m^{\prime}+\rho\right)}|u|_{s}|v|_{t}
$$

avec $\sum c_{q}^{2} \leq C$.

Deuxième cas : $\gamma=2$. Vu la localisation du support de $1-X$, avec un découpage spectral de $a_{q}(\cdot, \eta)$, de $b_{r}$ et $c_{r-\nu}$ on a :

$$
\begin{array}{r}
\left|\left(T_{q}^{(2)}(u, v)\right)(\zeta)\right| \leq \sum_{\substack{|\alpha| \leq M-1 \\
r \geq q+N}} \int h_{q, r, \alpha}(\zeta-\theta-\sigma, \theta+\sigma) \lambda_{q}(\theta+\sigma) \\
\times f_{r}(\theta) g_{r}(\sigma) d \theta d \sigma
\end{array}
$$

avec $f_{r}=2^{r\left(m_{1}-j\right)}\left|\hat{u}_{r}\right|, g_{r}=2^{r\left(m_{2}-\ell\right)} \sum_{|\nu| \leq N}\left|\hat{v}_{r-\nu}\right|$, et

$$
\begin{aligned}
& h_{q, r, \alpha}(\mu, \sigma)=\sum_{\substack{|\nu| \leq N, p \geq q \\
\left|\alpha_{1}+\alpha_{2}\right| \leq M-1}} \sum_{\beta_{1} \leq \alpha_{1}} 2^{q\left(m^{\prime}-i-\left|\beta_{1}+\alpha_{2}\right|\right)} \partial_{\sigma}^{\alpha_{1}-\beta_{1}}\left\{\left(\Delta_{p} a_{q}(x, \sigma)\right)\right. \\
&\left.\times\left|\hat{b}_{r}^{\left(\alpha_{1}\right)}\right|\left|\hat{c}_{r-\nu}^{\left(\alpha_{2}\right)}\right|+\hat{a}_{q}(\cdot, \sigma)\left|\left(\Delta_{p} b_{r}^{\left(\alpha_{1}\right)}\right)\right|\left|c_{r-\nu}^{\left(\alpha_{2}\right)}\right|+\left|\hat{b}_{r}^{\left(\alpha_{1}\right)}\right||| \Delta_{p} c_{r-\nu}^{\left(\alpha_{2}\right)} \mid\right\}
\end{aligned}
$$

d'où $\left\|h_{q, r, \alpha}(\cdot, \sigma)\right\|_{L^{1}} \leq 2^{q\left(m^{\prime}-i-|\alpha|\right)} 2^{-q(\rho-\operatorname{Inf}(i, j, \ell))}\langle\sigma\rangle^{m}$ d'où, par application du Lemme 2.1 on a :

$$
\left|T_{q}^{(2)}(u, v)\right|_{0} \leq c_{q} 2^{-q\left(s+t-(n / 2)-m-m_{1}-m_{2}-m^{\prime}+\rho\right)}|u|_{s}|v|_{t}
$$

avec $\sum c_{q}^{2} \leq C$ et ce pour tout $(s, t)$ tel que $s+t \geq m+m_{1}+m_{2}$.

Troisième cas : $\gamma=3$. On découpe spectralement $b_{r}$ et $c_{r} ; \mathrm{vu} \mathrm{le}$ support de $X$ on a :

$$
\left|\left(T_{q}^{(3)}(u, v)\right)(\zeta)\right| \leq \sum_{\substack{|\alpha|=M \\ r \geq q+N}} \int h_{q, r, \alpha}(\zeta-\theta-\sigma, \sigma) \lambda_{q}(\theta+\sigma) f_{r}(\theta) g_{r}(\sigma) d \theta d \sigma
$$

avec $f_{r}=2^{r\left(m_{1}-j\right)}\left|\hat{u}_{r}\right|, g_{r}=2^{r\left(m_{2}-\ell\right)} \sum_{|\nu| \leq N}\left|\hat{v}_{r-\nu}\right|$ et

$$
\begin{aligned}
& h_{q, r, \alpha}(\mu, \sigma)=\sum_{\substack{\beta_{1} \leq \alpha_{1},|\nu| \leq N \\
p \leq q+N, p^{\prime} \leq q+N}} \\
& 2^{q\left(m^{\prime}-i-\left|\beta_{1}\right|-\left|\alpha_{2}\right|\right)} \iint_{0}^{1}\left|\partial_{2}^{\alpha_{1}-\beta_{1}} \hat{a}_{q}\left(\mu-\xi^{\prime}-\eta^{\prime}, \sigma+t \xi^{\prime}\right)\right| \\
& \times\left|\xi^{\prime \alpha_{1}} \varphi\left(2^{-p} \xi^{\prime}\right) \hat{b}_{r}(\xi)\right| \times\left|\eta^{\alpha_{2}} \varphi\left(2^{-p^{\prime}} \eta^{\prime}\right) \hat{c}_{r-\nu}\left(\eta^{\prime}\right)\right| d \xi^{\prime} d \eta^{\prime} d t
\end{aligned}
$$

BULLETIN DE LA SOCIÉTÉ MATHÉMATIQUE DE FRANCE 
d'où on $a$ :

$$
\begin{aligned}
& \operatorname{Sup}_{\sigma \in C_{r}}\left\|h_{q, r, \alpha}(\cdot, \sigma)\right\|_{L^{1}} \\
& \leq 2^{q\left(m^{\prime}-i-|\alpha|\right)+r m} \sum_{\substack{p \leq q+N \\
p^{\prime} \leq q+N}} 2^{-p\left(\rho-j-\left|\alpha_{1}\right|\right)} 2^{-p^{\prime}\left(\rho-\ell-\left|\alpha_{2}\right|\right)}
\end{aligned}
$$

Si $\alpha_{1}=[\rho]-j+1: \alpha_{2}=i=\ell=0$ et $|\alpha|=[\rho]-j+1$

$$
\operatorname{Sup}_{\sigma \in C_{r}}\left\|h_{q, r, \alpha}(\cdot, \sigma)\right\|_{L^{1}} \leq 2^{r m} 2^{-q\left(\rho-m^{\prime}\right)}
$$

de même si $\alpha_{2}=[\rho]-\ell+1$.

Si $\alpha_{1} \leq[\rho]-j,\left|\alpha_{2}\right| \leq[\rho]-\ell$ on a :

$$
\operatorname{Sup}_{\sigma \in C_{r}}\left\|h_{q, r, \alpha}(\cdot, \sigma)\right\|_{L^{1}} \leq 2^{r m} 2^{q\left(m^{\prime}-i-|\alpha|\right)}
$$

d'où par une ultime application du LEMme 2.1 on a pour tout $(s, t)$ tel que $s+t \geq m+m_{1}+m_{2}$

$$
\left|T_{q}^{(3)}(u, v)\right|_{0} \leq c_{q} 2^{-q\left(s+t-(n / 2)-m-m_{1}-m_{2}-m^{\prime}+\rho\right)}|u|_{s}|v|_{t}
$$

avec $\sum c_{q}^{2} \leq C$ d'où le ThÉORÈme 2.2 .

\section{BIBLIOGRAPHIE}

[1] Alinhac (S.). - Évolution d'une onde simple. - Actes des journées E.D.P. de Saint-Jean-de-Monts, 1985 .

[2] Alinhac (S.). - Paracomposition et opérateurs paradifférentiels, Comm. Partial Differential Equations, t. 11, 1986, p. 87-121.

[3] Alinhac (S.). - Interaction d'ondes simples pour des équations complètement non linéaires. - Séminaire E.D.P. de l'Ecole Polytechnique, $1985-1986$.

[4] BEALS (M.). - Self spreading and strengh of singularities for solutions of semilinear wave equations, Ann. of Math., t. 118, 1983, p. 187-214.

[5] BEALS (M.). - Propagation of smoothness for non linear second order strictly hyperbolic differential equations, Proc. Sympos. Pure Math., t. 43, 1985.

[6] BONY (J-M.). - Calcul symbolique et propagation des singularités pour des équations aux dérivées partielles non linéaires,Ann. Sci. Éc. Norm. Sup., 1981, p. 209-246.

[7] BONY (J-M.). - Interaction des singularités pour des équations aux dérivées partielles non linéaires, Séminaire Goulaouic-Meyer-Schwartz, 1981-1982, $\mathrm{n}^{\circ} 2$.

[8] BONY (J-M.). - Second microlocalization and propogation for semi linear hyperbolic equations, dans Contribution to the workshop and symposium on hyperbolic equations and related topics, Katata and Kyoto, August 27 - Sept. 5,1984 , p. $11-50$.

TOME $116-1988-\mathrm{N}^{\circ} 3$ 
[9] Chemin (J-Y.). - Localisation des singularités pour les solutions d'équations aux dérivées partielles non linéaires en dimension 2 ou à l'ordre 1, C.R. Acad. Sci. Paris Sér. I Math., t. 303, 1986, p. 407-410.

[10] Chemin (J-Y.). - Calcul paradifférentiel précisé et applications à des équations aux dérivées partielles non semi-linéaires. - Préprint, à paraître au Duke Math. J.

[11] CoIfMAnn (R.) et MEYER (Y.). - Au-delà des opérateurs pseudodifférentiels, Astérisque, t. 57, 1978.

[12] Godin (P.). - Propagation of $C^{\infty}$ regularity for fully non linear second order strictly hyperbolic equations in two variables, Trans. Amer. Math. Soc., t. 290, 1985 , p. $825-830$.

[13] Melrose (R.) et Ritter (N.). - Interaction of non linear progressing waves I, Ann. of Math., t. 121, 1985, p. 187-213.

[14] Meyer (Y.). - Remarque sur un théorème de J.-M. Bony. - Rend. Circ. Mat. Palermo (1), 1981.

[15] Mizohata (S.). - Lectures on the Cauchy problem. - Bombay, Tata Institute, 1965 .

[16] RAUCH (J.) et REED (M.). - Non linear microlocal analysis of semi linear hyperbolic systems in one dimension space, Duke Math. J., t. 49, 1982, p. 397475.

BULLETIN DE LA SOCIÉTÉ MATHÉMATIQUE DE FRANCE 